\title{
Peter Auer \\ Genau! Der auto-reflexive Dialog als Motor der Entwicklung von Diskursmarkern
}

\section{Einleitung: das Powerpoint-Genau}

Es ist üblich, dass Studierende und jüngere Wissenschaftler/innen ihre Powerpoint-Präsentation mit dem Diskursmarker ${ }^{1}$ genau gliedern oder sogar einleiten. Genau schließt einen Abschnitt (Aktivität) ab und leitet zugleich den nächsten Abschnitt (Aktivität) ein (letztere kann auch die Präsentation selbst sein); es hat also eine für „Gliederungssignale“ (Gülich 1970) typische Scharnierfunktion:

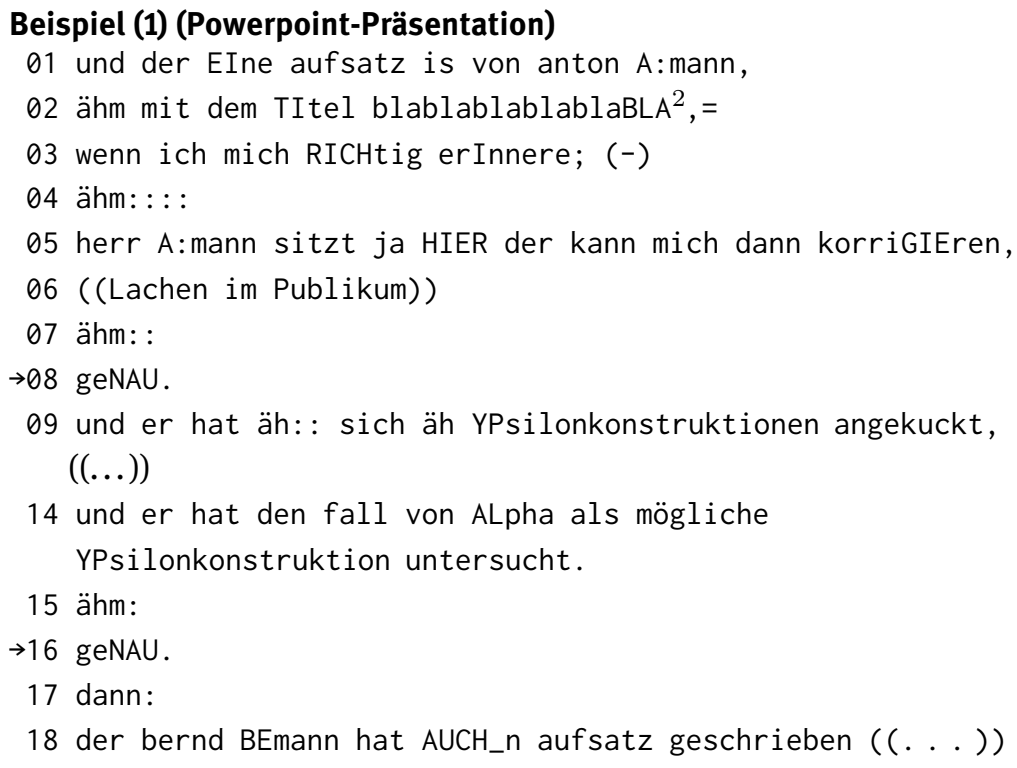

1 Der Begriff wird hier in einem weiten Sinn gebraucht, der auch alleinstehende „Diskurswörter“/ „Antwortwörter“ (etwa Bestätigungs- und Negationswörter) einschließt. Fast alle „Antwortwörter“ können auch in der Satzperipherie verwendet werden, so dass eine klare Trennung kaum möglich erscheint. Wie später zu zeigen sein wird, ist genau überdies gerade kein Antwortwort. 2 Die Titel der im Ausschnitt verwendeten Zeitschriftenaufsätze und ihre Themen sowie die Autorennamen wurden aus Anonymisierungsgründen unkenntlich gemacht. 
Der häufigste Verwendungstyp ist in Z. $16 \mathrm{zu}$ beobachten: der Sprecher kommt (anhand seiner Power-Point-Folie) zu seinem ,nächsten Punkt‘, in diesem Fall dem nächsten Literaturtitel in einer Aufzählung bisheriger Forschung zum Thema seines Vortrags. Der Übergang wird von einem Blick auf die Folie begleitet, auf die dadurch auch die Aufmerksamkeit der Zuhörer/Zuschauer gelenkt wird. Voran geht eine Häsitationspartikel (ähm in Z. 07). Die Besprechung des nächsten Literaturtitels wird mit dann eingeleitet (Z. 09). Genau ist hier ganz offensichtlich nicht responsiv-zustimmend gemeint; es bestätigt keinesfalls die Mitteilung/Behauptung, dass ,Anton Amann einen Aufsatz über die Ypsilonkonstruktion geschrieben' hat, denn diese Behauptung hat der Präsentierende ja selbst produziert.

Der Sprecher verwendet genau aber noch ein zweites Mal, nämlich in Z. 08; wieder geht eine Häsitationspartikel voraus. Hier ist der Diskursmarker nicht das Scharnier zwischen zwei gleichrangigen inhaltlichen Abschnitten, die linear abgearbeitet werden, sondern steht am Ende einer kleinen Seitensequenz mit dem Publikum und dem anwesenden Autor Anton Amann (Z. 04-06), nach der der Sprecher - eingeleitet mit und - wieder zur Besprechung des Aufsatzes von Anton Amann zurückkehrt. Die Struktur ist sehr ähnlich:

$$
\begin{aligned}
& \ddot{a} h m \text { - genau - dann... (Z. 16) } \\
& \ddot{a h h m} \text { - genau - und... (Z. 08) }
\end{aligned}
$$

Noch offensichtlicher als im vorher besprochenen Fall bezieht sich genau hier nicht auf den Inhalt der vorherigen Äußerung, sondern leitet die Rückkehr in die Rahmenaktivität (PP-Präsentation) ein.

Dieses Powerpoint-Genau, wie man es nennen könnte, führt bei älteren Sprechern und Sprecherinnen des Deutschen immer noch zu Irritationen, während es für jüngere völlig unproblematisch ist. Dies deutet auf sprachlichen Wandel hin. Offenbar hat sich genau zu einem Gliederungssignal entwickelt, während ältere Sprecher/innen damit noch eine pragmatische (Zusatz-)Bedeutung verbinden (eine bestimmte Art von Zustimmung), die sie situativ unpassend finden. Im Folgenden will ich versuchen, diesen Wandel zu rekonstruieren.

Viele Verwendungsweisen von genau - nicht jedoch das Power-Point-Genau sind schon recht ausführlich beschrieben worden (jüngst von Oloff 2016, 2017, aber u. a. auch schon von Werlen 2010 und Willkop 1988: 138-146). Die Ergebnisse dieser Arbeiten können - mit einigen Ergänzungen - verwendet werden, um die Entwicklung von genau zu modellieren und mit anderen Diskursmarkern zu vergleichen. Im Zentrum steht dabei die Frage nach den Mechanismen, die über den Einzelfall hinaus für den pragmatischen Wandel von Diskursmarkern eine gewisse Bedeutung beanspruchen können. Zwei solche Mechanismen sollen vor allem 
betrachtet werden. Einer ist für Diskursmarker typisch: eine responsive Partikel wird zu einer übergangsmarkierenden oder sogar TCU-einleitenden (oder umgekehrt). Der Mechanismus ist nicht nur bei der Entwicklung von genau im Spiel, sondern auch bei Diskursmarkern wie gut, klar, sicher, schön, ok u. a. Bei dieser Gruppe von Markern hat sich aus der responsiven Verwendung als alleinstehende Bestätigung eine weitere, TCU-initiale Verwendung im Rahmen von konzessiven Strukturen entwickelt. Es bietet sich also ein Vergleich an, zumal diese Gruppe von Markern (trotz insgesamt deutlicher Unterschiede in ihrer Verwendungsweise) bei responsiver Verwendung mit genau in ihrer Funktion überlappt, dem vorherigen Gesprächsteilnehmer zuzustimmen. Der zweite Mechanismus besteht darin, dass der Sprecher einen internen Dialog externalisiert. Er spricht also quasi zu sich selbst (als Rezipient eigener Gedanken oder Handlungen), lässt aber den oder die anderen Interaktionsteilnehmer (als Beobachter des Geschehens, bystander) an diesem inneren Dialog teilhaben. ${ }^{3}$ Ich nenne das den auto-reflexiv dialogischen (oder kurz: autoreflexiven) Gebrauch.

\section{Von der Gradpartikel zum Diskursmarker}

Wenn wir uns mit Diskursmarkern, ihrer Entstehung und ihrem Wandel beschäftigen, verlassen wir den Bereich der traditionellen Grammatik, denn wir reden weder über die Struktur von Sätzen noch über die Struktur von Wörtern, sondern über die Struktur von Turns und die Frage, ob bestimmte Wörter oder Ausdrücke in bestimmten Turn-Positionen ihren präferierten Ort finden, also von etwas, was Schegloff (1996) als „positional sensitivity of grammar“ bezeichnet (vgl. Deppermann i.d. B. und mit Bezug auf Diskursmarker auch Heritage/Sorjonen 2018). Obwohl man die Entstehung von Diskursmarkern als einen Fall der Sedimentierung sprachlicher Praktiken (also im weitesten Sinn als Grammatisierung im Bereich der Makro- oder Diskurssyntax) verstehen kann (vgl. Auer/Günthner 2005), wird sie teilweise von anderen Prinzipien bestimmt als die klassischen Grammatikalisierungsprozesse (im engen Sinn der Entstehung neuer grammatischer Kategorien, vgl. auch Linke i.d. B.). Ein offensichtlicher Unterschied ist, dass Diskursmarker in der Regel aus Mitgliedern anderer Wortklassen entstehen; ein weiterer, dass diese im Lauf des Prozesses, in dem sie sich zu Diskursmar-

\footnotetext{
3 Dass der Sprecher selbst es ist, der seine eigenen Handlungen oder Gedanken kommentiert, heißt natürlich nicht, dass solche Äußerungen nicht auch mit dem bystander im Blick produziert werden und sich sekundär auch an diesem Rezipienten zweiter Ordnung orientieren können. Vgl. zu den komplexen Formen der Rezipienz zuletzt Deppermann/Schmidt (2016).
} 
kern entwickeln, nicht etwa stärker in paradigmatische und syntagmatische Strukturen eingebunden werden (wie das aus der klassischen Grammatikalisierungsforschung bekannt ist, vgl. Lehmann 1985/2002), sondern sich aus diesen lösen. Sie werden an der Peripherie der Satzstruktur zu relativ autonomen Sprachmitteln. Wenn man die Entstehung von Diskursmarkern trotzdem als eine bestimmte Form der Grammatisierung sehen kann, dann deshalb, weil die Bedeutung der Wörter auf diesem Weg an Semantik verliert und an Pragmatik gewinnt.

Der responsive Diskursmarker genau wird meist auf die homophone Gradpartikel zurückgeführt (König 1991: 17), die selbst von einem lokalen Adjektiv nouw durch Abstraktion von dessen ursprünglicher Bedeutung (,Nähe') gebildet wurde. Dieses genau hat sich aus dem Satzzusammenhang gelöst, was man sich wohl am ehesten durch Verkürzung von Sätzen wie das ist genau so, das ist genau richtig, etc., vorstellen kann.

In seiner responsiven Verwendung kann der Marker heute allein (aber auch oft mit ja kombiniert) einen Redebeitrag bilden, mit dem der Sprecher der vorausgehenden Meinungsäußerung einer anderen Gesprächsteilnehmerin ,voll und ganz` zustimmt:

\section{Beispiel (2) ,Stichworte“ (Callhome deutsch)}

((Telefongespräch über ein eineinhalbjähriges Kind))

$01 \mathrm{~B}$ : und dann hat er gesagt paPIER,

02 also er braucht jetzt paPIER zum SCHREIben.

$03 \mathrm{~A}$ : wo?

04 B: auf den

05 spricht nicht in SÄTzen sondern nur in STICHworten.

$06 \mathrm{ja.}$

07 A: aha.

$08 \mathrm{~B}:$ ja wei ${ }^{2}$ er ist ja erst eineinHALB.

09 A: ja, aber SCHAU mal;

10 mit eineinhalb jAhren norMAlerweise

können die kinder doch nur PApa sagen oder so,

$\rightarrow 11$ B: ja geNAU.

12 also das war richtig GOLdig.

13 das ist doch allerHAND;=ne,

Dieses responsive genau kann auch durch ganz verstärkt werden: 


\section{Beispiel (3) ,Ehrlichkeit' (BB)}

((Es geht um die Frage, wie man neue Freunde gewinnt.))

01 Jhn: also (-) ick DENke,

02 ähem wenn_wa schon davon REden,

03 FREUNde zu gewinnen,

04 denk ich der WICHtigste punkt is natürlich (-) die EHRlichkeit?

$\rightarrow 05 \mathrm{Jrg}:<<\mathrm{p}>$ GANZ genau. $>$

$06 \mathrm{Jhn}$ : so wat $(-)$ entWICkelt sich halt irgendWIE und dat- (-)

07 ick glaub auch $\mathrm{NICH}$,

08 dass man das auf_n PUNKT bringen kann;= ja,

In Beispiel (2) hat B. von einem eineinhalbjährigen Kind erzählt, das zum Malen nach ,Papier‘ verlangt, dabei aber keinen ganzen Satz verwendet, sondern in ,Stichworten' spricht. A. gibt zu bedenken, dass auch die Einwortäußerung $\mathrm{Pa}$ pier für einen Eineinhalbjährigen schon eine ordentliche Leistung ist, denn andere Kinder würden in diesem Alter nur Papa sagen. Dieser Einschätzung stimmt B. mit genau (Z.11) ,voll und ganz‘ zu.

In Beispiel (3) beschreibt John, welche Dinge in einer Freundschaft wichtig sind. Er beginnt damit, dass ,Ehrlichkeit‘ der ,wichtigste Punkt‘ sei. Diese moralische Aussage wird von Jürgen mit GANZ genau quittiert (Z. 5). Er schließt sich dadurch Johns Meinung, voll und ganz‘ an.

Auch Handlungsvorschläge können mit genau angenommen werden:

\section{Beispiel (4), Shakespeare' (BB63)}

((Andrea und Verena müssen den Text für eine Theateraufführung lernen. Die folgende Sequenz spielt sich beim Frühstück ab.))

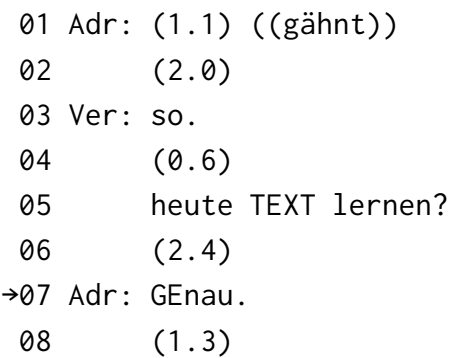

Verena beginnt die Sequenz beim Frühstück mit einem fallend intonierten so. (Z.03), das eine neue Aktivität einleitet (Stukenbrock 2010). Diese wird darauf mit einer deontischen Infinitivkonstruktion (Deppermann 2007) beschrieben: heute TEXT lernen?. Nach einer Pause stimmt Andrea mit GEnau zu. Genau könnte in 
diesem sequenziellen Zusammenhang nicht verwendet werden, wenn eine Handlungsoption erst neu eingeführt würde, also der zustimmenden Teilnehmerin vorher gar nicht bekannt war. Wer nach einem Vorschlag mit genau antwortet, drückt aus, dass ihm die vorgeschlagene Handlungsoption ebenfalls schon durch den Kopf gegangen ist und dass sie zumindest im common ground der Teilnehmer verankert (wenn auch nicht aktualisiert) war. Dies unterscheidet genau von einer einfachen Zustimmungspartikel wie ok oder ja. (Klar hätte hingegen die zusätzliche Wirkung zu signalisieren, dass der Vorschlag überflüssig war, weil die vorgeschlagene Handlung sowieso selbstverständlich ist.)

Neben der einfachen Zustimmung zu Meinungen oder Vorschlägen hat genau also auch eine epistemische Bedeutungskomponente: Die nächste Sprecherin gibt zu verstehen, dass der Sachverhalt, den der Sprecher ihr mitgeteilt hat, Teil ihres eigenen Wissens ist. Die in Frage stehende Proposition ist nicht erst durch die Äußerung des vorherigen Sprechers in den Wissensbestand der bestätigenden Sprecherin übergegangen, sondern war ihr schon vorher und unabhängig von der Äußerung des Anderen bekannt. Deshalb ist genau nicht für die Rezeption von Neuigkeiten geeignet. In diesem sequenziellen Kontext würde eine Erwiderung mit genau ebendiesen Handlungstyp retrospektiv ungültig machen: die Rezipientin würde signalisieren, dass sie schon weiß, was ihr als Neuigkeit mitgeteilt wird, die Neuigkeit also keine Neuigkeit ist.

Oloff (2017) bezeichnet genau deswegen als Intersubjektivitätsmarker und untersucht verschiedene konversationelle Formate, die diese Analyse unterstützen, in denen also genau dem vorherigen Sprecher signalisiert, dass der Inhalt seiner Äußerung richtig, aber auch schon bekannt ist. Ein solches Format sind Rückversicherungsfragen (confirmation requests), mittels derer eine Teilnehmerin eine Proposition vom anderen Teilnehmer bestätigt bekommen möchte, der zu diesem Wissen privilegierten Zugang hat. Genau kommt hier in der dritten Position vor, also für die Bestätigung:

\section{Beispiel (5) ,vierundzwanzig، (DOMIAN 9-6-2000)}

((Viktoria ist die neue Anruferin, die gerade in der Leitung ist und in der Phone-inShow begrüßt wird.))

08 D0: vikTOr[ia v=VIErundzwanzig das stimmt ja?

09 VI: $\quad[a ̈ h m ;$

$\rightarrow$ GEn[au; (.) vierundZWANzig bin ich.

11 DO: [SEHR gut; (-) SEHR gut;

12 um was GEHTS denn bei dir.

Domian hat bereits aus der Regie mitgeteilt bekommen, dass seine nächste Anruferin vierundzwanzig Jahre alt ist, möchte diese Information aber von ihr selbst 
bestätigt bekommen (Z. 08) und auf diese Weise auch dem Publikum zugänglich machen (vgl. sein VIErundzwanzig das stimmt ja? in Z.8). Viktoria kommt dieser Bitte um Bestätigung in Z. 10 durch genau nach, gefolgt von der Wiederholung der Altersangabe.

Die Altersinformation kommt in diesem Fall aus zweiter Hand und ist daher bestätigungsbedürftig. Im folgenden Beispiel beruht sie hingegen auf einer Inferenz: Sandra vermutet, dass Annes Italienischunterricht in der Kursstufe 1 (,K1') begonnen hat. Diese Vermutung wird Anne zur Bestätigung vorgelegt:

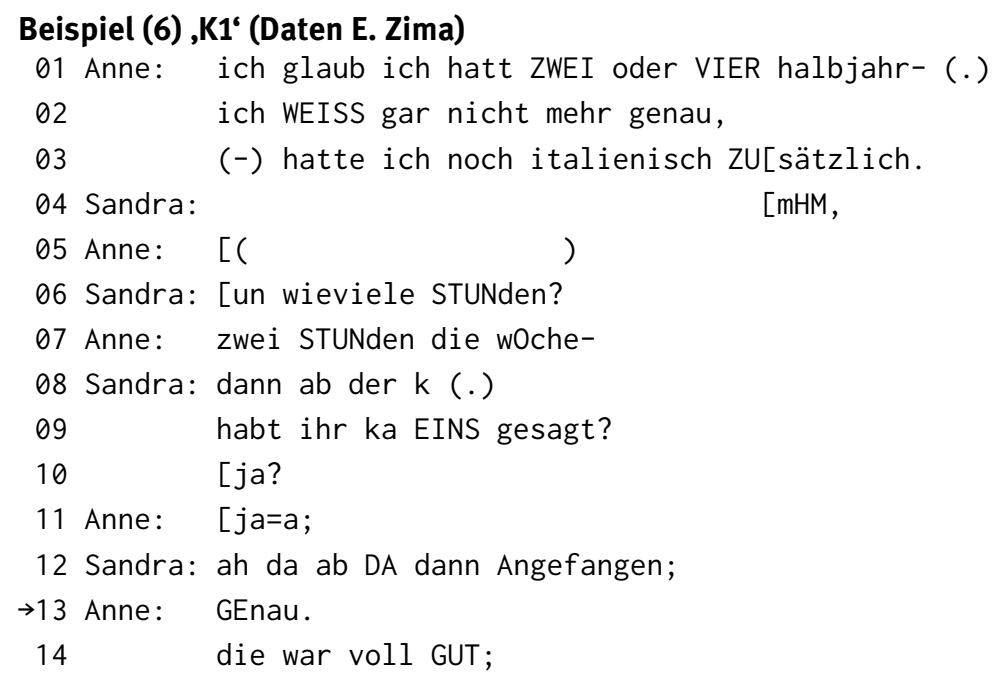

Anne berichtet, dass sie in der Schule ,zwei oder vier Halbjahre“ Italienischunterricht hatte. Sandra vermutet (beginnend in Z. 08, unterbrochen von einer Rückfrage nach der korrekten Bezeichnung der Kursstufe, abschließend in Z. 12), dass dieser Unterricht in der Oberstufe erteilt wurde. Anne bestätigt diese Vermutung mit GEnau.

Bitten um Bestätigung präsupponieren eine Wissensasymmetrie zwischen dem Befragten und dem Fragenden; jener hat privilegierten Zugang zu dem Wissensbestand, nach dem dieser fragt. Genau markiert die Übereinstimmung der Wissensstände der beiden Teilnehmer.

Recht ähnlich lässt sich genau als Responsivpartikel im Rahmen von Ko-Konstruktionen analysieren (vgl. Brenning 2015, Günthner 2015, Oloff 2016). Hier führt die zweite Teilnehmerin die begonnene TCU des ersten Teilnehmers zu Ende, oft eingeladen durch Pausen oder andere Häsitationsmarker im emergierenden Turn des ersten Sprechers. Jede ko-konstruierende Vervollständigung greift aber in das Rederecht dieses Sprechers ein und legt ihm bestimmte Worte (oder Inhalte), in 
den Mund'. Nur er weiß, ob sie seinen Intentionen entsprechen. Auf der Bühne der Interaktion kann der erste Sprecher diese Entsprechung bestätigen und damit die Übereinstimmung seiner Intention mit der ko-konstruierten Turn-Vervollständigung markieren:

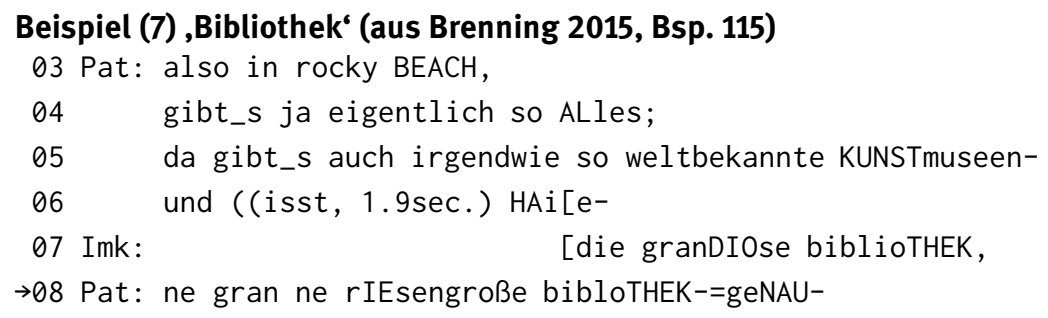

Beispiel (8) ,Salz' (aus Brenning 2015, Bsp. 135)

57 Est: hey aber LAHR,

59 ja LAHR,

$60 \quad(-)$

61 hat (-) he he he [nicht genu:]g ähm [ähm]

62 Lin: [he LAHR, ]

63 Sar: $[$ SALZ, ]

$\rightarrow 64$ Est: $<<:-)>$ SALZ genau->

65 Sar: die ham [JEdes jahr irgendwie ni]ch genug SALZ;

66 Est: [für die STRASsen- ]

In Ausschnitt (7) zählt Patrizia die Vorteile des Lebens in Rocky Beach auf. Die Liste beginnt mit den ,Kunstmuseen“ (Z. 04-05) und wird mit den ,Haien“ (Z. 06) fortgeführt. Das dritte ${ }^{4}$ Listenelement trägt Imka bei: die ,grandiose Bibliothek`. Patrizia muss zu dieser Listenvervollständigung Stellung nehmen. Sie stimmt mit genau und einer etwas abgewandelten Formulierung (bei identischer Referenz: ,ne riesengroße Bibliothek') zu. Imka hat zwar vielleicht nicht den Pluspunkt von Rocky Beach genannt, den Patrizia selbst in die Liste aufnehmen wollte, Patrizia stimmt aber zu, dass die ,riesengroße Bibliothek ${ }^{`} \mathrm{zu}$ den Dingen gehört, die das Leben in Rocky Beach angenehm machen. Die Vervollständigung war in ihrem

\footnotetext{
4 Es lässt sich vermuten, dass Imka mit der Ko-Konstruktion auf die Pause in Patrizias Turn vor HAie reagiert, während der sie mit dem Essen beschäftigt ist; Imka scheint schon bei der Produktion des zweiten Listenelements zu zögern. Obwohl die biblioTHEK an der Oberfläche der Interaktion als drittes Listenelement erscheint, ist sie, wie die Überlappung mit HAie zeigt, als zweites geplant.
} 
Sinn, was durch genau ausgedrückt wird. Die Ko-Konstruktion hat eine epistemische Seite (beide Sprecherinnen geben zu erkennen, dass sie die Bibliothek von Rocky Beach kennen und wissen, dass sie sehr groß ist), aber auch eine evaluative: die Bibliothek wird von beiden als Teil der Liste von Dingen akzeptiert, die das Leben an diesem Ort auszeichnen.

In Ausschnitt (8) beginnt Esther einen von Lach- und Zögerungspartikeln durchzogenen Redebeitrag, der die Stadt Lahr zum Thema hat: ,Lahr hat nicht genug... ‘. An dieser Stelle greift Sarah in den emergenten Redebeitrag ein und vervollständigt ihn durch das Nomen ,Salz‘. (Gemeint ist Streusalz.) Die Vervollständigung ist in gewisser Weise durch Esthers doppelte Häsitation nach genug in Z. 61 lizensiert. Wieder ist zwar nicht offensichtlich, dass Esther exakt diese Aussage machen wollte; sie akzeptiert jedoch Sarahs Vervollständigung so, als ob sie sie ihren Intentionen entsprochen hätte, indem sie sie wiederholt und durch genau bestätigt (Z. 64). In diesem Fall ist die Funktion des Diskursmarkers rein epistemisch: Esther bestätigt, dass ihr Wissen über den Winterdienst der Stadt Lahr dem von Sarah entspricht.

Schließlich lässt sich die Rolle von genau als epistemischem Intersubjektivitätsmarker auch aus selbstinitiierten Fremdreparaturen belegen. In diesem Fall lädt ein augenblicklicher Sprecher die anderen Teilnehmer ein, ihm bei der Behebung einer Formulierungsschwierigkeit (oft beim Finden des richtigen Worts oder Namens) zu helfen. Vorschläge, dieses Problem zu überwinden, müssen vom Sprecher dann abgelehnt oder angenommen werden. Letzteres passiert im folgenden Beispiel:

\section{Beispiel (9) ,Merida“ (Daten E. Zima)}

01 Anne: pochahOntas ist die EINzige disney prinzessin die ein tatTOO hat;

$((\ldots))$

10 Anne: hab ich neulich geLEsen.

11 Sandra: UND (.) ich hab AUCH gelesen-=

12 es kam doch EINmal dieser;

13 WIE heißt die;

14 die mit den krAssen roten HAAren?

15 Mario: arIELLE,

16 Anne: me(.)RINda,

17 Sandra: ja: :

18 [meRIda oder so>-

[((zeigt mit dem Finger auf Anne))

19 Anne: [meRIda>-

[((zeigt mit Finger auf Sandra)) 
$\rightarrow 20$ Sandra: geNAU;

und DA hieß es irgendwie dass die_s dass die die erste DISney heldin wär; (.) ((etc.))

Nachdem Anne berichtet hat, sie habe gelesen, dass Pochahontas die erste Disney-Heldin mit einem Tattoo sei, möchte Sandra ihrerseits berichten, sie habe gelesen, Merida sei die erste weibliche Heldenfigur von Disney gewesen. Dies gelingt ihr aber nicht ohne fremde Hilfe, denn es fehlt ihr der Name der Disney-Heldin. Sie unterbricht daher ihren emergierenden Redebeitrag und fragt ihre beiden Gesprächsteilnehmer explizit nach dem Namen der Disney-Figur ,mit den krassen roten Haaren'. Marios Vorschlag $\operatorname{arIELLE~(Z.~15)~wird~übergangen,~Annes~Vor-~}$ schlag me(.)RINda hingegen aufgegriffen und bestätigt (Z. 17: ja::), allerdings dabei leicht verändert und mit einem Approximationsmarker versehen (Z. 18: meRIda oder so). Im nächsten Schritt liefert Anne die Version meRIda als sicheres Wissen; ihr meRIda in Z. 19 ist also nicht einfach eine Wiederholung von Sandras approximativem meRIda: der korrekte Name ist ihr in diesem Augenblick eingefallen (was sie durch eine Zeigegeste auf Sandra deutlich macht). Sandra bestätigt ihrerseits den Namen durch genau (Z. 20) und setzt ihren Turn fort. Genau markiert die Deckung der beiden Wissensbestände; Sandra signalisiert damit nicht etwa, dass sie Anne glaubt, sondern dass sie weiß, dass sie recht hat, weil sie sich selbst wieder erinnert. Ihr Wissen stammt nicht von Sandra, sondern aus ihrer eigenen Erinnerung.

Insgesamt lässt sich festhalten, dass der Weg von der Gradpartikel zum Diskursmarker mit einem semantisch-pragmatischen Wandel verbunden ist, der die ursprüngliche Semantik i.S. v. ,exakt` in eine gut definierte pragmatische Funktion verwandelt: Genau signalisiert die Übereinstimmung der Meinungen und Absichten, öfter aber des Wissens der Beteiligten. Dabei wird diese Übereinstimmung nicht als eine dargestellt, die in der Interaktion entsteht, sondern als eine, die schon vorgängig da war. Obwohl der respondierende Teilnehmer in zweiter Position spricht, hat er nur „exakt das geäußert [...], was der Sprecher selbst hätte sagen können“ (Willkop 1988: 140).

Genau überlappt also semantisch-pragmatisch zwar mit anderen Diskursmarkern wie gut, schön, ok oder einfachem ja, denn sie alle können ebenfalls Zustimmung ausdrücken. Aber nur genau präsentiert diese Zustimmung so, dass der zustimmende Teilnehmer suggeriert, diese Übereinstimmung habe ,immer schon existiert.

Wie auch bei anderen zustimmenden Diskursmarkern $\mathrm{zu}$ beobachten, verwenden manche Sprecher genau allerdings positionell so undifferenziert, dass eine funktionale Ausbleichung und ein Übergang zum Rezeptionsmarker zu beob- 
achten ist. ${ }^{5}$ Rezeptionsmarker bestätigen nur die Rezeption, nicht den Inhalt der Äußerung oder Äußerungskomponente des Sprechers. Sie geben dem Sprecher auf diese Weise das Signal weiterzusprechen (continuer). Das lässt sich daran erkennen, dass sie auch innerhalb emergenter Redebeiträge an Stellen vorkommen, an denen der Sprecher zögert und/oder die Rezeption einer TCU-internen Einheit (etwa einer referierenden Nominalphrase) bestätigt haben möchte. Im folgenden Beispiel versuchen drei Freundinnen die (Vor-)Geschichte von Luke Skywalker aus ,Starwars‘ zu rekonstruieren:

\section{Beispiel (10) ,Luke Skywalker‘ (Daten E. Zima)}

((Emma hat zwar alle alten und neuen Starwars-Filme gesehen, ist sich aber nicht sicher, dass sie die genealogischen Zusammenhänge noch versteht - wer ist der Vater von wem? Jana und Lena kennen sich noch weniger aus, sind aber mit den alten Starwars-Filmen vertraut.))

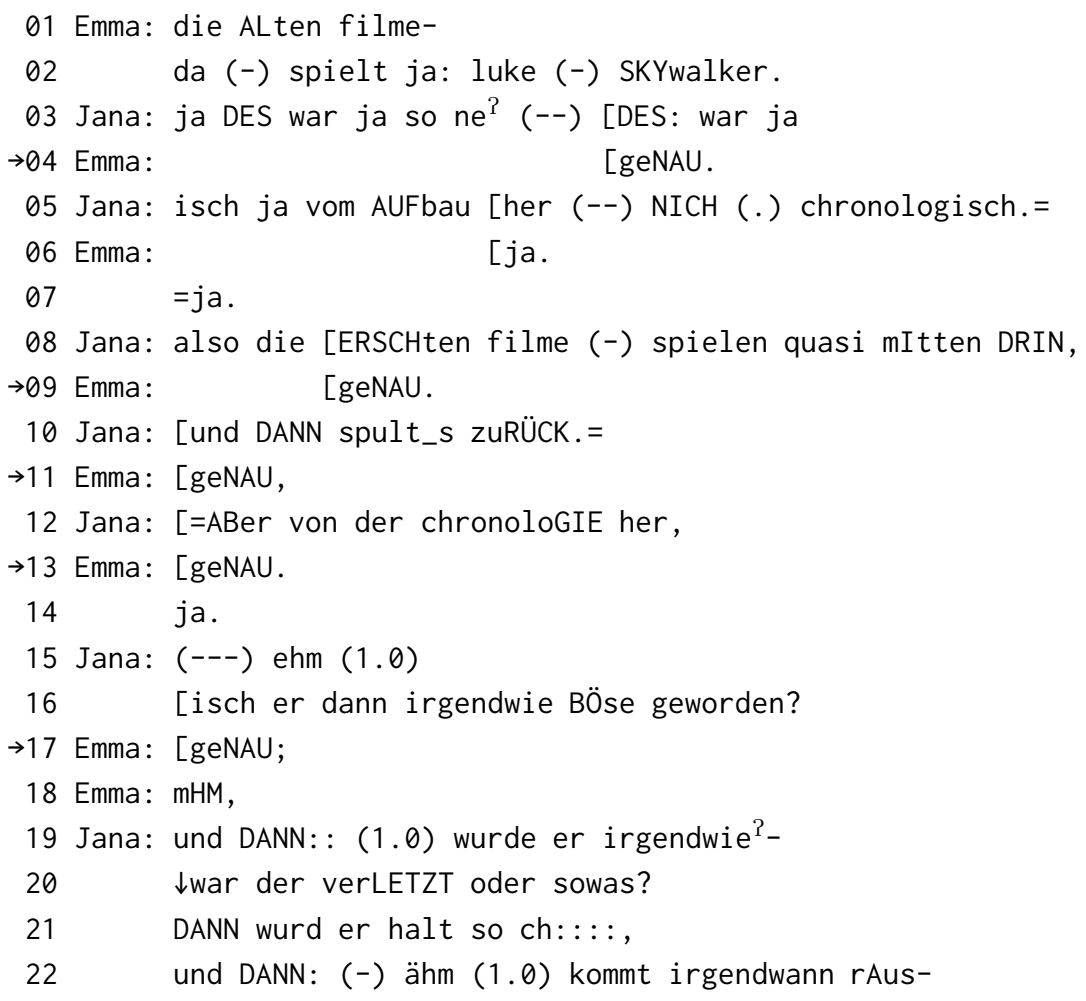

5 Werlen (2010: 351) findet diese Ausbleichung schon im Freiburger Corpus aus den 1970er Jahren, wobei seine Kriterien für die Einstufung allerdings eher intuitiv bleiben. 


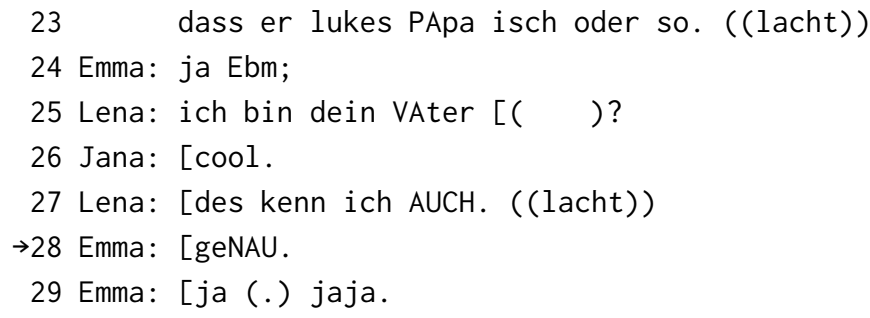

Emma, die Starwars-Spezialistin, hat kurz vorher gestanden: „ob ich jetzt so GANZ den zusammenhang verstanden hab weiss ich nich. (.) also; WER (-) jetzt von WEM der vater isch, (-) und wer dann DESwegen (-) von WEM dann wieder das KIND (.) und der ENkel is, (-) hab ich NICH so ganz verstanden." Nun beginnt Jana zusammenzutragen, was sie selbst über die Geschichte weiß (Z. 03-25). Emma reagiert auf die einzelnen Elemente dieser Rekonstruktion mit verschiedene Diskursmarkern, darunter genau, aber auch einfaches ja. Meist sind sie am Ende von propositionalen Einheiten positioniert und intersubjektivieren das Wissen von Jana. Emma ist aufgrund ihres Spezialistinnenstatus dazu berechtigt: Sie signalisiert durch genau, dass Emmas Wissen mit ihrem eigenen übereinstimmt und daher korrekt ist. Es gibt aber drei Fälle, in denen genau nicht so interpretiert werden kann. Sowohl in Z. 04 als auch in Z. 09 und Z. 17 platziert Emma ihr genau an Stellen, an denen Jana noch keine vollständige Proposition formuliert hat (nach ja DES war ja so ne ${ }^{\text {? }}$ in Z. 03, nach also die in Z. 08 und nach ABer von der chronoloGIE her, ehm in Z. 12/15). ${ }^{6}$ In diesen Fällen ist eine präzise Zuordnung des genau zu bestimmten Aussagen der Sprecherin nicht mehr möglich; obwohl Emma pauschal der Richtigkeit der Beschreibungen Janas zustimmt, lässt sich diese epistemische Übereinstimmung nicht mehr konkret auf bestimmte Komponenten der Äußerung beziehen.

\section{Vom responsiven Intersubjektivitätsmarker zum Gliederungssignal, Teil 1: auto-reflexive Verwendungen}

Genau als Diskursmarker im heutigen Deutsch hat sich aber über diese sequenziell-responsive Funktion hinaus weiterentwickelt und dient neben der hetero-dia-

6 Dasselbe gilt für Platzierungen von ja in Z. 06 und Z. 14, das hier ebenfalls nur Rezeptionssignal ist. 
logischen auch der auto-dialogischen Bestätigung. Hier stimmt sich der Sprecher in einem Dialog mit sich selbst zu, der allerdings in der Interaktion externalisiert und damit den anderen Teilnehmern zugänglich gemacht wird. Auch diese Funktion von genau ist der bisherigen Forschung nicht entgangen und wird schon von Willkop (1988) unter dem Stichpunkt, eigenreferenzieller Gebrauch“ beschrieben.

Oloff (2017) weist darauf hin, dass sich der auto-reflexive Gebrauch von genau vor allem zeigt, wenn der Sprecher ein mentales Problem zu bewältigen hat. Das kann z. B. eine Wortsuche oder ein problematischer Prozess des Sich-Erinnerns innerhalb der Produktion eines Turns sein, wie etwa im folgenden Beispiel:

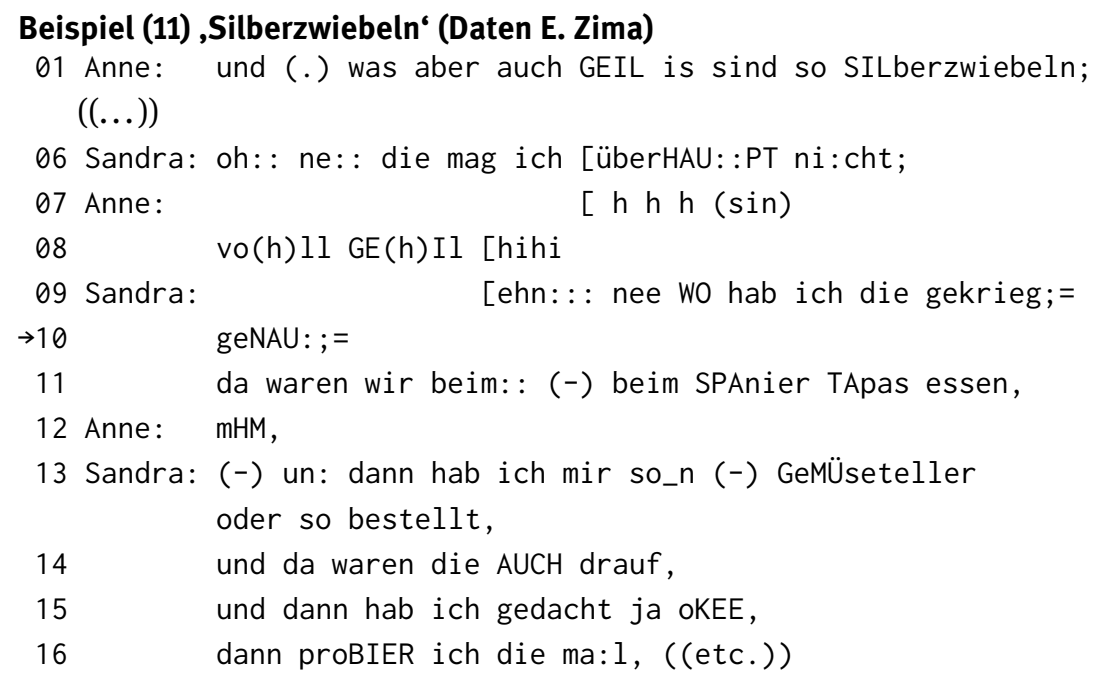

Sandra hat ihren Widerwillen gegen ,Silberzwiebeln' zum Ausdruck gebracht (Z. 06) und versucht sich nun zu erinnern, auf welches Restaurant-Erlebnis dieser Widerwillen zurückgeht. Der mentale Prozess wird durch die an sich selbst gerichtete Frage wo hab ich die gekrieg;=, (Z. 09) in der Interaktion sichtbar. Sandra bricht die Artikulation dieser an sich selbst gerichteten Frage vor dem zu erwartenden auslautenden Plosiv / $t$ / in gekriegt (Z. 09) mit genau ab. Sie markiert durch den Diskursmarker den genauen Zeitpunkt der Erinnerung und damit den exakten Übergang in einen anderen epistemischen Status (nämlich vor dem letzten Laut des Wortes gekriegt). Der Prozess der Erinnerung an die ersten Silberzwiebeln wird also als Dialog der Sprecherin mit sich selbst inszeniert. ${ }^{7}$

7 Genau wäre deshalb in solchen autodialogischen Fällen, in denen Fragen der Erinnerung bearbeitet werden, mit ach so austauschbar, das ebenfalls einen plötzlichen Erkenntnisgewinn markieren kann (vgl. zu diesem Diskursmarker in Reparatursequenzen Golato/Betz 2008). 
Ganz ähnlich im folgenden Ausschnitt, in dem allerdings die auto-reflexive Frage fehlt:

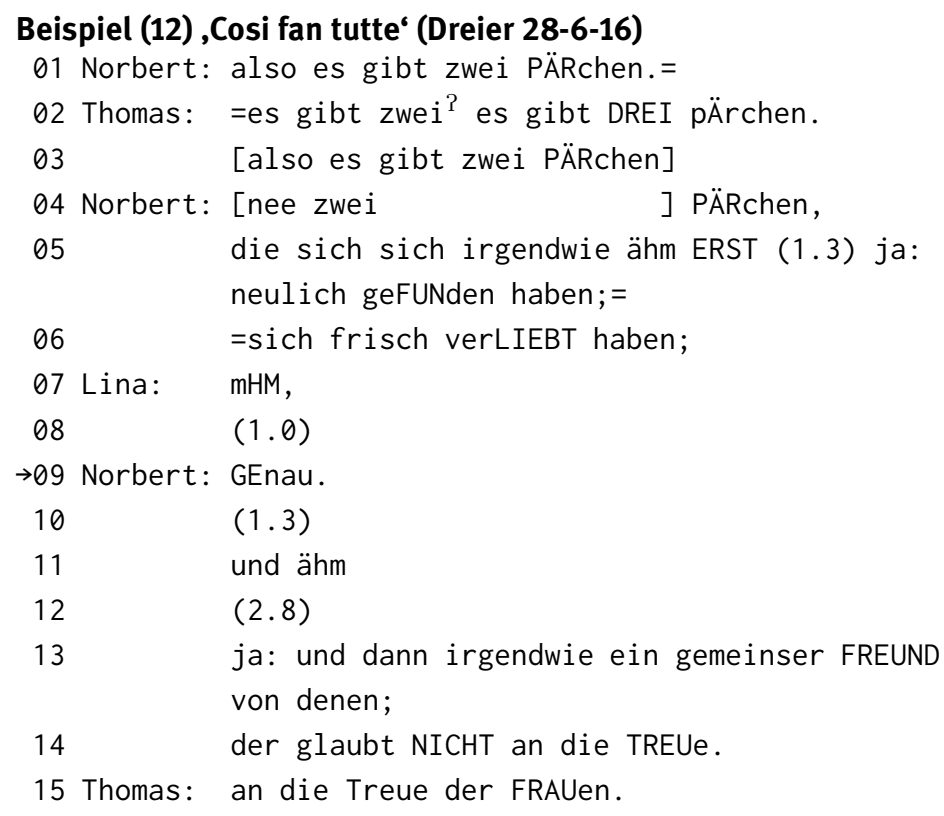

Norbert und Thomas versuchen hier Lina den Inhalt der Oper ,Cosi fan tutte“ zu erzählen, die sie vor kurzem gesehen haben. Norbert, der Haupterzähler, wird gleich zu Beginn von seinem Freund Thomas korrigiert, als er die Protagonisten als „zwei Pärchen“ einführt; es seien ja drei Pärchen. Beide fangen nun an, den Widerspruch aufzulösen, aber Norbert setzt sein Rederecht durch und präzisiert, dass es zwei Pärchen gebe, die sich gerade verliebt hätten (04-06). Nach einem Rezipientensignal der Adressatin Lina (Z. 07) entsteht eine längere Pause, während der Norbert offenbar versucht, sich an die weiteren Details zu erinnern oder diese zu ordnen. Diese mentalen Prozesse werden durch genau in Z. 09 als abgeschlossen präsentiert. Allerdings dauert es noch weitere 1,3 Sekunden, bevor der Sprecher tatsächlich mit seiner Formulierung beginnt, und auch dieser Versuch stockt zunächst (Z.13), bevor der fünfte Protagonist (Don Alfonso) eingeführt wird.

Genau kann also immer verwendet werden, wenn ein Sprecher ein kognitives Problem selbst beseitigt $\mathrm{zu}$ haben vorgibt. Es ist responsiv, reagiert allerdings nicht auf ein interaktives Objekt (wie den Redebeitrag eines anderen Teilnehmers), sondern auf einen nur diesem Sprecher direkt zugänglichen mentalen Vorgang. Daraus ergibt sich der kritische Kontext für die Weiterentwicklung 
des Diskursmarkers zu einem Gliederungssignal ohne diese autoreflexiv responsive Funktion: die kognitive Leistung, auf die genau reagiert, ist für die übrigen Interaktionsteilnehmer (oder uns als Analysierende) nicht mehr ohne Weiteres zugänglich und kann bestenfalls erschlossen werden kann. Noch mehr als in (11) und (12) trifft das auf das folgende Beispiel zu:

\section{Beispiel (13) ,Schauspieler` (BB)}

((Die Kamera zeigt die im Inneren des Containers für die Aufführung eines Shakespeare-Stücks probenden Bewohner. Andrea liest in einem Shakespeare-Drama; Jürgen sitzt ihr gegenüber, bereit, mit ihr an der Memorierung des Textes zu arbeiten))

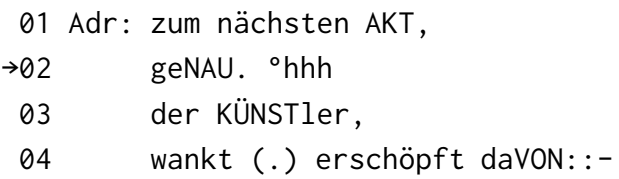

Andrea leitet den Übergang zum Einüben des nächsten Akts in Zeile 01 ein. Es folgt ohne weitere Verzögerungen, die auf mentale Problemlösungsversuche hindeuten könnten, unmittelbar der Diskursmarker geNAU. Dazwischen scheint eine kognitive Leistung zu liegen, die Andrea lesend erbringt, die aber weder für Jürgen noch für uns präzise identifizierbar ist: Möglicherweise hat sie die relevante Textstelle gefunden. Die Sprecherin scheint sich selbst zu bestätigen. Aber für die anderen Teilnehmer ist der Anlass für diese Bestätigung nicht mehr sichtbar. Was zu bestätigen ist, liegt außerhalb des interaktionalen Raums. Die Beziehung zwischen Bestätigungsobjekt und Bestätigung geht verloren. Sichtbar ist nur, dass die Sprecherin einen neuen Abschnitt einleitet und dafür eine kognitive Leistung erbringen musste. Dem anderen Interaktionsteilnehmer eröffnet sich daher die Möglichkeit, genau als reines Gliederungssignal zu reinterpretieren. Auf diese Weise kann sich der autodialogische Bestätigungsmarker zum Gliederungssignal weiterentwickeln.

In den folgenden beiden Beispielen wird diese funktionale Entleerung des selbst-responsiven genau offensichtlich. Im ersten Beispiel spricht die Anruferin bei ,Domian“ über die Entmündigung ihrer alkoholkranken und dementen Mutter:

\section{Beispiel (14), Vormund' (DOMIAN)}

$\rightarrow 01$ VI: sie ist durch diese klInik geNAU entMÜNdigt worden?=

02 und [dann halt erst_n n_FREMder halt,

03 DO: $\quad[j a$,

$04 \mathrm{VI}$ : und jetzt hat mein VA:ter sogar den den vormund endlich,

$05{ }^{\circ}$ hhh [(dann hat mein) vater ${ }^{\text {? }}$ 
06 D0: $\quad$ [WAS hat der $v$ was hat der VAter?

$\rightarrow 07 \mathrm{VI}$ : mein (.) geNAU mein vater ist jetzt der VORmund.=

08 D0: [ist der VoRmund ah ja (.) ja.

$\rightarrow 09$ VI: [=also er ist der (--) geNAU.

Von den drei Verwendungen von genau in diesem Ausschnitt entspricht nur die letzte (in Z. 09) dem in Abschnitt 2 besprochenen Muster (genau als Erwiderung auf eine Bitte um Rückbestätigung, d.h. mit Bezug auf Z. 08). Genau in Zeile 01 und erneut in 07 kann nur autoreflexiv interpretiert werden. Weder will die Sprecherin sagen, dass die Klinik ihre Mutter ,exakt‘ entmündigt hat, noch, dass ihr Vater ,exakt' der Vormund ihrer Mutter ist. Genauso wenig dient genau der Sprecherin dazu, einer Äußerung ihres Gesprächspartners zustimmen. Genau wird vielmehr parenthetisch von der Sprecherin in ihren Redebeitrag eingeschoben. Es scheint, als kommentiere die Sprecherin ihre eigene kognitive Leistung des Erinnerns, als ob sie kurz vergessen hätte, worum es eigentlich geht und es nun bestätigen müsste, ohne dass ein solches Erinnerungsproblem an der Oberfläche der Interaktion sichtbar geworden wäre.

Im zweiten Fall stellt sich eine Studentin ihren beiden Kommilitoninnen vor:

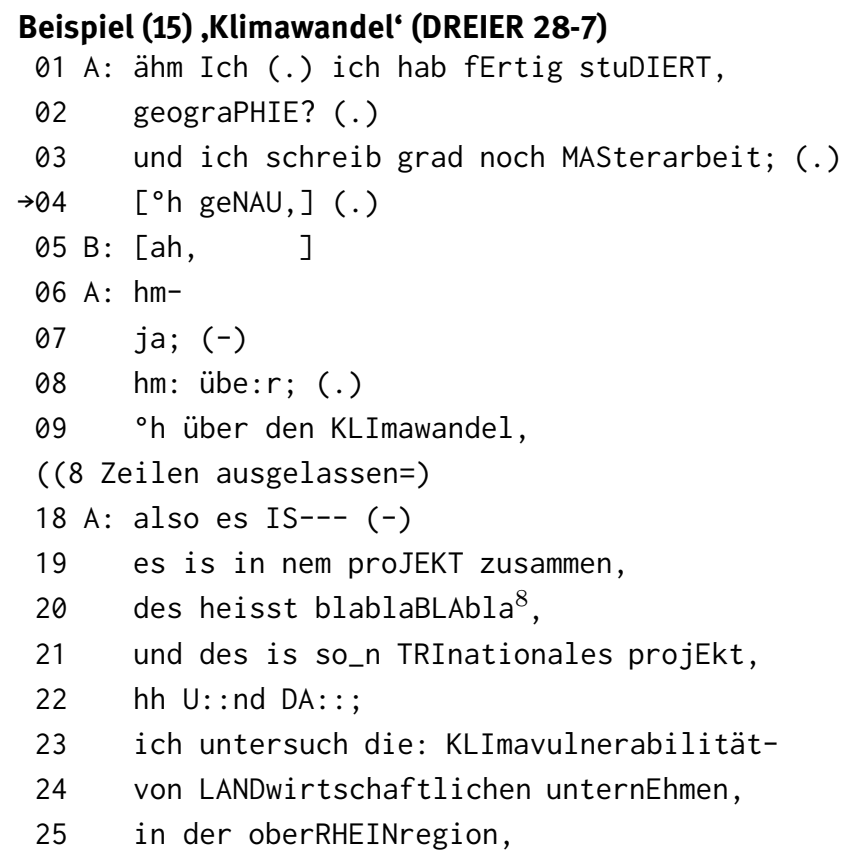

8 Name aus Anonymisierungsgründen gelöscht. 
26 B: HM;

$\rightarrow 27$ A: geNAU;

28 also ich führ exPERteninterviews du:rch;

29 befrag sie zu HANdlungsstrategien;

$(($ etc. $))$

Sprecherin A. hat in den ersten drei Zeilen einen abgeschlossenen Redebeitrag geliefert, in dem sie ihr Studienfach und ihre augenblickliche Tätigkeit (Arbeit an der Master-Arbeit) beschrieben hat. B. geht darauf mit einem kurzen Rezeptionssignal ein (Z. 05: ah). Zur gleichen Zeit produziert A. ein genau, dessen Bezug nicht erkennbar ist: es gibt kein sichtbares mentales Problem, das bewältigt wurde. Ebenso wenig kann es sich um eine Zustimmung handeln: Eine Sprecherin kann nicht die Übereinstimmung ihres Wissens mit dem Inhalt ihrer eigenen Äußerung markieren. Es folgt nun eine Expansion, in der zunächst das Thema der Masterarbeit mitgeteilt wird (,über den Klimawandel‘, Z. 09) und anschließend (nachdem die Rezipientin in den ausgelassenen Zeilen kommentiert hat, dass es sich dabei doch um ein sehr generelles Thema handele) das Projekt weiter erläutert wird (Z. 18-25). Wieder beschränkt sich die Rezipientin auf ein Rezeptionssignal (Z. 26). Anschließend produziert die Sprecherin ein weiteres genau. Sich selbst zuzustimmen, wäre auch hier unsinnig; erneut ist auch kein kognitives Problem zu erkennen. Es liegt für den Hörer nahe, den Marker als Abschluss der TurnErweiterung zu verstehen; da er aber dem continuer folgt, durch den B. ja A. schon zum Weitersprechen eingeladen hat, ist er auch Einleitung für die erneute Weiterführung des Themas, die ab Z. 28 erfolgt. In beiden Fällen wird genau also als Gliederungssignal reinterpretierbar.

Autoreflexiv-dialogische Selbstbestätigungen sind als interaktionales Phänomen nicht auf genau beschränkt. Eng verwandt ist das turnbegleitende (und vor allem turnabschließende) selbstbestätigende Nicken, das man vor allem bei amerikanischen Sprecherinnen des Englischen, zunehmend aber auch bei Deutschen beobachtet. Die Sprecherin gliedert durch dieses Signal (das sonst als Bestätigung oder zumindest Rezeptionssignal vom Gesprächspartner eingesetzt wird) ihren eigenen Redebeitrag. Ohne dass auf dieses Phänomen hier genauer eingehen zu können, soll hier lediglich ein illustratives Beispiel der Verdeutlichung dienen:

Beispiel (16) (https://www.youtube.com/watch?v=dUmldCClbTE; 18.08.2020) ((Alexandria Ocasio-Cortez on The Daily Show with Trevor Noah/veröffentlicht 29/7/18/letzter Zugang 2/1/20; HN = headnod))

$01 \mathrm{AOC}$ : and so a lot of what we need to do is

[reprioritize ]

[drei schnelle, kleine HNs ] 
(1.0) WHAT we want to [accomplish (.) as a NAtion.]

[vier schnelle, kleine HNs ]

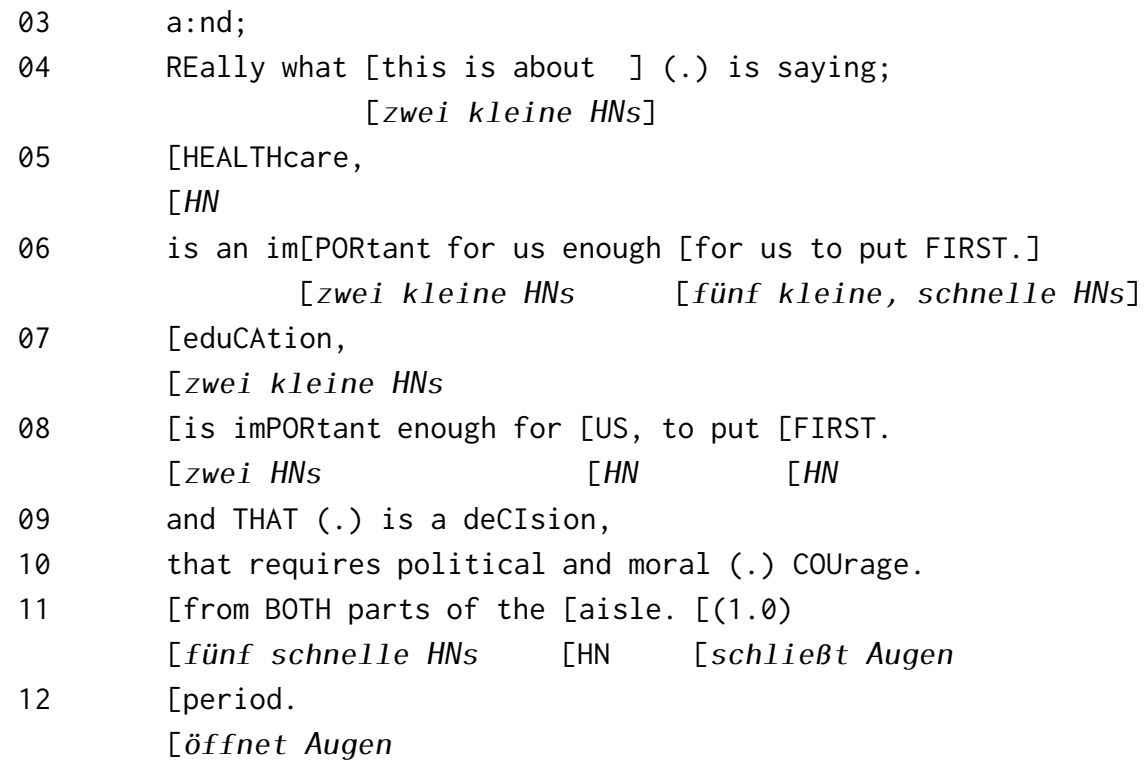

13 Host: [thank you so much for being yourself.

Dies ist ein (vermutlich einstudiertes) politisches Statement der demokratischen Nachwuchspolitikerin, mit dem sie ihren Besuch in der Talkshow von Trevor Noah abschließt. Die Produktion des Turns wird von zahlreichem Kopfnicken begleitet. Die letzte IP (Z. 11) wird als solche durch sechsmaliges Nicken in Kombination mit dem Schließen der Augen und dem verbalen Abschlussmarker period markiert.

Redebegleitendes Nicken während einer Meinungsäußerung ist in vielerlei Hinsicht dem deutschen auto-dialogischen genau ähnlich. Beide gehen auf eine Praktik zurück, die ursprünglich sequenziell-responsiv ist und Zustimmung signalisiert. (Daneben hat Nicken natürlich auch die Funktion eines Rezeptionssignals.) In beiden Fällen steht die sequenziell-responsive (hetero-dialogische) Verwendung aber heute neben einer auto-dialogischen. ${ }^{9}$

\footnotetext{
9 Ein Unterschied zu genau liegt darin, dass das Sprecherinnennicken keine Bewältigung eines kognitiven Problems indiziert, sondern wohl vor allem die Zustimmung des Rezipienten evozieren möchte, der zur Kopie dieses Nickens eingeladen wird.

Der autoreflexive Dialog ist auch in zahlreichen anderen Fällen ein Mechanismus, der für den pragmatischen Wandel von Bedeutung ist. Der Übergang von der responsiven in die turn-intern konzessive Verwendung der Diskursmarker gut oder $o k$ ist ein Beispiel dafür (siehe dazu den nächsten Abschnitt). Ein anderes Beispiel ist die Verwendung des Anredepronomens $d u$ als Selbst-Referenz des Sprechers auf sich selbst (wie in Auer/Stukenbrock 2018 beschrieben).
} 


\section{Vom Intersubjektivitätsmarker zum Gliederungssignal, Teil 2: positionelle Aspekte}

Die auto-reflexiv dialogische Verwendung von genau ist auch bei Power-Point-Präsentationen wie in Beispiel (1) anzutreffen, denn schließlich handelt es sich hier um eine monologische (multimodale) Gattung (vgl. Günthner/Knoblauch 2007; Knoblauch i.d. B.), die die dialogische Verwendung als Intersubjektivitätsmarker ausschließt. Wie die anderen im letzten Abschnitt besprochenen Verwendungsweisen im konversationellen Kontext indiziert dieses Power-Point-genau manchmal noch die selbstständige Bewältigung einer kognitiven Herausforderung durch den Sprecher oder lässt sich zumindest so interpretieren. Diese kann zum Beispiel im Überfliegen der neuen, gerade angeklickten Folie oder auch im (stillen) Lesen des nächsten Punkts auf einer Folie bestehen, der nun vorgetragen werden soll. Solche Fälle kann man noch als (auto-)responsiv interpretieren (vgl. etwa Z. 16 in Ausschnitt (1)). Die Vortragenden versichern sich selbst, dass sie über die kognitiven Voraussetzungen für die Bewältigung des nächsten Schritts verfügen.

Nicht immer ist aber eine solche Interpretation möglich. Dann liegt die Reinterpretation als reines Gliederungssignal ohne zustimmende Bedeutungskomponente nahe (wie in Z. 08 in Ausschnitt (1)). Als Gliederungssignal mit Scharnierfunktion leitet genau eine nächste Aktivität oder einen nächsten Abschnitt ein und schließt zusätzlich den vorherigen ab, ist also sowohl vorwärts- wie auch rückwärtsgewandt; und oft scheint sogar die vorwärtsgewandte Seite zu überwiegen. Um diese positionelle Reanalyse von genau von der responsiven zur überleitenden Funktion ohne Zustimmungskomponente geht es in diesem Abschnitt.

Ihr Ursprung ist in der auto-reflexiv dialogischen Verwendung schon angelegt. Betrachten wir die dafür analysierten Beispiele noch einmal, so zeigt sich, dass zur responsiven immer auch eine weiterweisende Komponente gehört. Denn wenn ein Sprecher sein kognitives Problem bewältigt hat, ist zu erwarten, dass er nun die Handlung, der dieses Problem entgegenstand, durchführen wird. So ist von Sandra zu erwarten, dass sie, nachdem sie sich an das Restaurant erinnert hat, in dem sie ,Silberzwiebeln` gegessen hat, nun dieses Ereignis erzählt:

\section{Beispiel (17) ,Silberzwiebeln“}

09 Sandra: ehn:: 
Genau bestätigt hier nicht nur, dass der Erinnerungsprozess erfolgreich abgeschlossen ist, es leitet auch in die Erzählung vom Restaurantbesuch über. Dasselbe gilt für Ausschnitt (13), wo Adriana bereits angekündigt hat, dass sie sich dem ,nächsten Akt‘ widmen möchte. Sobald das - wie auch immer geartete - kognitive Hindernis, um diese Absicht in die Tat umzusetzen, beseitigt (und dies durch genau den anderen mitgeteilt) wurde, ist die Durchführung dieser Handlung zu erwarten:

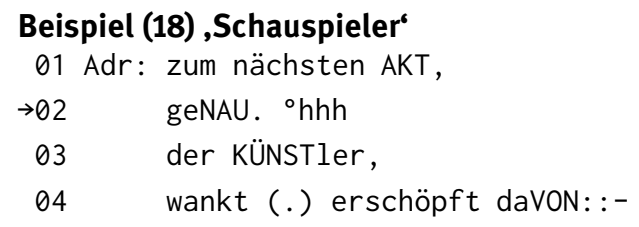

Drastisch wird das verdeutlicht, wenn genau einen Redebeitrag einleitet, ohne sich responsiv auf den Betrag des vorherigen Sprechers zu beziehen:

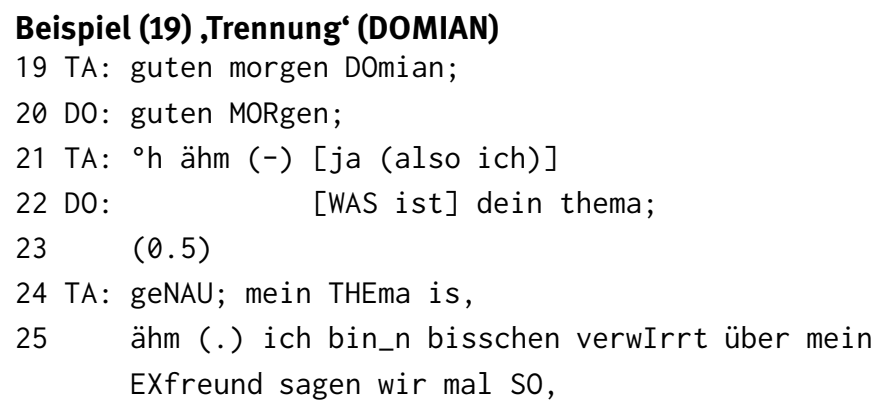

In diesem Beispiel Antwortet TA in Z.24 auf die Frage Domians nach ihrem Problem (Grund für den Anruf im Studio). Sie beginnt ihre Antwort mit genau. Dieses genau drückt weder Zustimmung zu einem Vorschlag oder Übereinstimmung mit dem Wissen des anderen Gesprächsteilnehmers aus, noch indiziert die Anruferin damit, dass sie ein kognitives Problem bewältigt hat. Es dient vielmehr der Einleitung in die Gesprächsphase der Problemdarstellung. Ein Rest an retrospektiver Orientierung bleibt in diesem Beispiel lediglich dadurch enthalten, dass möglicherweise ein interaktives Problem als erledigt dargestellt wird, nämlich die TurnTaking-Turbulenz, die durch den vorzeitigen Start der Problemdarstellung unmittelbar nach dem initialen Grußaustausch (Z.21) entstanden ist, also noch vor der Aufforderung durch den Moderator, ihr Problem darzustellen. Es kommt dadurch zu einer Phase des Simultansprechens (Z. 21, 22), einer Störung des Gesprächsablaufs, die mit genau abgeschlossen wird. 
Keinerlei Bezugspunkt hat genau hingegen in Ausschnitt (15), hier wiederholt als Beispiel (20), was sich u. a. daran zeigt, dass genau in Z. 05 mit B.s Rezipientensignal überlappt und ihm in Z. 27 sogar folgt:

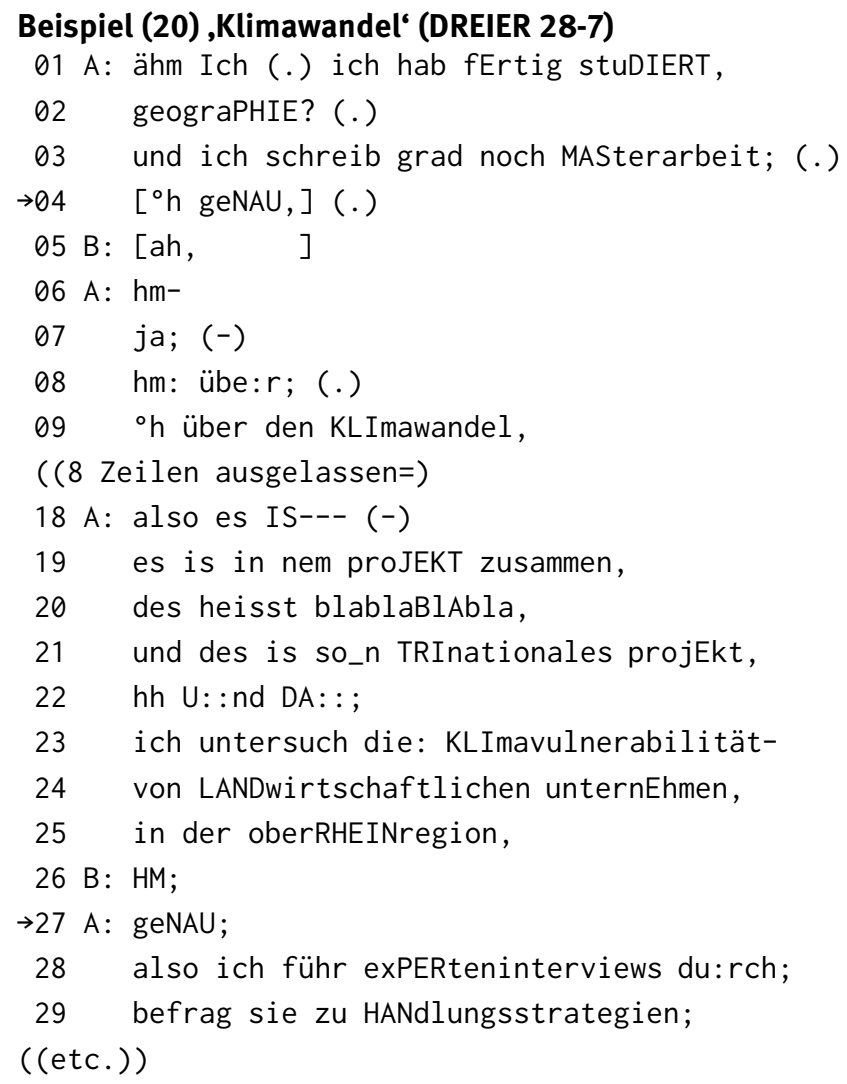

Mit dem zunehmenden Verlust seines auto-reflexiven Bezugspunkts in einem (mentalen) Problem verliert genau seine responsive Komponente immer mehr. Solche Abschwächungen der Reponsivität sind nicht auf genau beschränkt. Auch andere zustimmende Diskursmarker wie klar, gut, schön, ok können ihre responsive Position verlassen (vgl. Auer/Lindström 2016; vgl. auch ähnlich zu initialem und zwar bei Günthner 2012). Allerdings verläuft die Entwicklung dieser zuletzt genannten Gruppe von affirmativ-responsiven Markern anders als bei genau. Ich beschränke mich auf einige Anmerkungen zu gut.

Alleinstehendes, responsives gut hat je nach sequenziellem Kontext unterschiedliche Funktionen. Sein Ursprung ist die Verwendung als evaluierendes Adjektiv, die man etwa in Erwiderungen auf Neuigkeiten findet. Hier drückt gut eine 
positive Bewertung dieser Neuigkeit - insbesondere aus der Perspektive des Erzählers - aus:

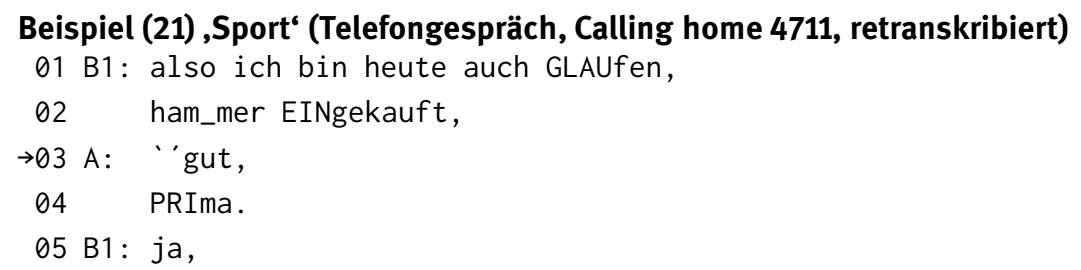

\section{Beispiel (22) ,Zimmerhock' (Telefongespräch, Calling Home 5097, retranskri- biert)}

$01 \mathrm{~A}$ : jetzt br- muss ich nicht mehr zur english STUdy hall geh:n,

02 und kann abends kann ich auch wenn ich lUst hab FERNsehn kucken oder so was-

$\rightarrow 03$ B: [igu: :t.

$03 \mathrm{~A}$ : [und muss nicht immer in meinem ZIMmer hock ${ }^{\text {}}$ bleiben?=un: $\mathrm{d}^{\text {h }}$

04 ja aber das ist eGAL; ( (etc.))

Die Sprecherin A. in (21) findet es positiv, dass der Gesprächspartner, wie er ihr mitgeteilt hat, ,heute laufen war‘ (die Bewertung ist indirekt ein Lob an ihn), die Sprecherin B findet es gut, dass ihr Gesprächspartner ,nicht mehr in die English Study Hall' gehen muss. In beiden Fällen ist die Prosodie von gut markiert, einmal durch die fallend-steigende Intonationsbewegung, das andere Mal durch Höherlegung und Dehnung.

Meist fehlt gut aber eine solche prosodische Markierung; er wird mit einfacher, fallender Tonhöhenbewegung produziert. Zugleich tritt die bewertende Funktion zurück oder fehlt ganz. Dagegen tritt die Funktion als Gliederungssignal in den Vordergrund, das eine Aktivität (Sequenz) abschließt und eine andere einleitet. Der Unterschied wird deutlich, wenn man die letzten beiden Beispiele mit dem folgenden vergleicht (das bereits als Beispiel (5) diskutiert wurde). Hier beendet Domians sehr gut die Einleitungsphase des Gesprächs; aber er bewertet dadurch nicht die dort gegebene Information (nämlich dass die Anruferin 24 Jahre alt ist). (Sehr) gut hat sich vollständig seine ursprüngliche Semantik als Adjektiv verloren und fungiert ausschließlich als Übergangsmarker; ${ }^{10}$ es ist in

10 Einen ähnlichen Fall beschreiben Antaki et al. (2000) für das Englische. Sie beobachten, dass markiert positive Bewertungen (wie brilliant und excellent) in institutioneller Kommunikation typischerweise von Seiten des Institutionenvertreters verwendet werden. Sie drücken keineswegs der Bewertung der vorherigen Aussage des Klienten aus (etwa der Antwort auf eine Frage), son- 
diesem Sinn nicht mehr responsiv, sondern sowohl rückwärts- als auch vorwärtsgewandt.

\section{Beispiel (23) ,vierundzwanzig' (DOMIAN 9-6-2000)}

((Viktoria ist die neue Anruferin, die gerade in der Leitung ist und in der Phone-inShow begrüßt wird.))

08 D0: vikTOr[ia v=VIErundzwanzig das stimmt ja?

09 VI: $\quad[$ ähm;

10 GEn[au; (.) vierundZWANzig bin ich.

$\rightarrow 11$ DO: [SEHR gut; (-) SEHR gut;

12 um was GEHTS denn bei dir.

Gut kommt aber nicht nur responsiv als Bewertung nach Mitteilungen/Neuigkeiten vor, es wird auch verwendet, um Handlungsoptionen (die beide Interaktionspartner betreffen) zu akzeptieren. Hier übernimmt gut die Funktion der Zustimmung. Auch in diesem Fall wirkt gut zugleich sequenzterminierend: Wer dem Vorschlag eines anderen zustimmt, schließt damit die Sequenz ab. Im folgenden Beispiel stimmt Andrea einem Vorschlag Johns zu, wie sie eine riesige Plastikkuh mit dem Spitznamen ,Big Else‘ bemalen könnten:

\section{Beispiel (24) ,Big Else“ (BB97)}

$01 \mathrm{Adr}$ : ich hatte ERST noch überlegt vielleicht noch das big BROTher logo [irgendwo aufzumalen; ]

$02 \mathrm{Jrg}$ :

$$
\text { [(das ); ] }
$$

03 Jhn: [det war ICke;]

04 [det kann ick vorne MAChen, ](0.5)

$05 \mathrm{Jrg}$ : [das KANN ich nich. ]

06 Jhn: aber dass big ELse druff steht. (-)

$07 \mathrm{Adr}$ : [achso ja.]

$08 \mathrm{Jrg}$ : [hä=Ḧ̈, ((zustimmend))]

$\rightarrow 09$ Adr: [gut.

10 Jhn: [aber jeNAUso wie det] LOgo.=

11 =so als KETte; =weißte?

$\rightarrow 12$ Adr: ja,=GUT; =

13 dann MACH das.

dern markieren den Übergang von einem zum nächsten Punkt in der institutionellen Agenda. Im Deutschen wird auch schön so verwendet. 
$14 \mathrm{Jrg}$ : [ich hatte das $\mathrm{AUCH}$; eh $\mathrm{AUCH}$ vor aber das krich ]

$15 \mathrm{Adr}$ : [weil das würd ich schon SCHÖN finden dass wir aus dem] [wir aus dem:-

$16 \mathrm{Jrg}$ : [aber das krich ich EH nich hin.

17 deswegen hab ich das direkt wieder verwoRfen.

18 Adr: ja dann MACH das doch.

19 Jhn: big ELse; weißte?=

$20 \mathrm{Jrg}: \mathrm{hm}:[\mathrm{:}$,

21 Adr: [ja; oKAY.

22 Jhn: ja aber jenau [wie das Logo schreiben.]

$23 \mathrm{Adr}$ [ [wie das lo-]

24 wie das LOgo.

$\rightarrow 25$ gut.

26 Jhn: hä_HÄ, ((zustimmend))

Andrea führt die Idee ins Gespräch ein, das ,Big Brother Logo irgendwo (auf die Plastikkuh) aufzumalen' (Z. 01); John hatte offenbar bereits eine ähnliche Idee und schlägt vor, den Spitznamen der Plastikkuh (,Big Else`) aufzubringen (Z. 04/06), eine Idee, die sowohl Andrea (achso, ja) als auch der dritte Teilnehmer, Jürgen (hähä,) überzeugt. Andrea versucht die Sequenz mit gut (Z. 09) abzuschließen, während John sie überlappend erweitert (Z. 10), indem er ankündigt, den Namen in der Gestaltung des Big-Brother-Logos aufzumalen. Andrea versucht erneut einen Sequenzabschluss mit ja, gut, (Z. 12) und dann mach das. Die Sequenz wird allerdings erneut erweitert, diesmal von Jürgen, der ergänzt, dass er dieselbe Idee bereits als (für ihn) undurchführbar verworfen hat, und simultan von Andrea selbst, die ihre Zustimmung zu diesem Plan zu begründen versucht. Ein zweites ja, dann MACH das doch in Z. 18 reiteriert den vorherigen Abschlussversuch. Noch ein letztes Mal wird die Sequenz von John erweitert, der seinen Plan von Big Else im Big-Brother-Design erläutert. Andrea wiederholt sein wie das LOgo bestätigend, um die Sequenz dann endgültig mit gut abzuschließen (Z. 25).

Andrea verwendet eine Reihe von Zustimmungssignalen; neben gut gehören dazu auch ja und $o k$. Typisch ist dabei die Reihenfolge: gut folgt ja (und okay), und wird seinerseits von dann mach das (doch) gefolgt.

07 ach so ja, 09 gut.

12/13 ja, gut; dann MACH das.

$18 \mathrm{ja}$, dann MACH das doch.

21 ja; okay. 25 gut. 
Dies legt die Interpretation nahe, dass gut über die reine Zustimmung zu Johns Plan hinausgeht (die auch bereits durch ja geleistet worden ist) und die spezifische Funktion hat, den Sequenzabschluss voranzutreiben.

Noch weiter von einer positiven Bewertung entfernt und noch näher an der sequenzterminierenden Verwendung des Markers ist das folgende Beispiel, in dem gut einen langen Streit zwischen Sabrina und Andrea abschließt:

\section{Beispiel (25) ,Vorhang' (BB86)}

((Der Sequenz geht ein Streit zwischen den beiden Frauen um die Verwendung eines Vorhangs voraus. Nachdem sich die Wogen geglättet haben, endet die Sequenz wie folgt:))

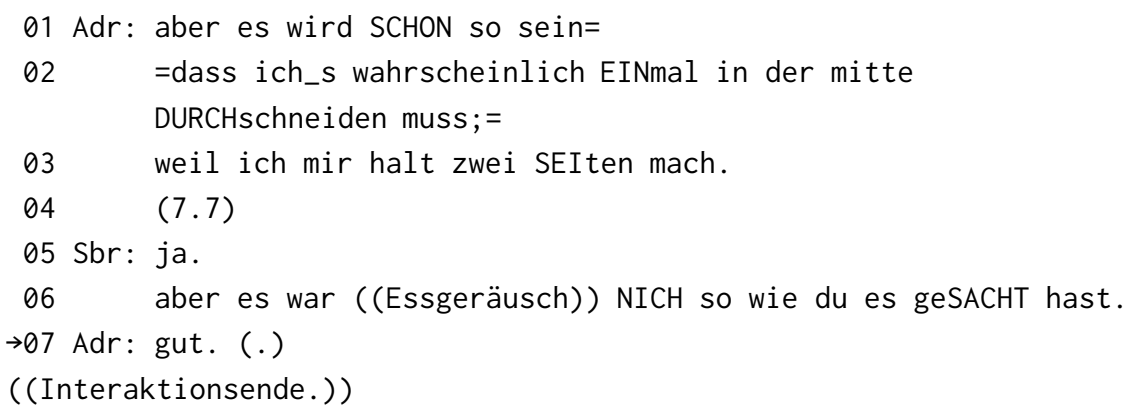

Nachdem sich die beiden darauf geeinigt haben, dass Andrea den Vorhang verwenden darf (vgl. Sabrinas ja in Z. 05), wird der Streit von Sabrina noch metakommunikativ nachbearbeitet. Sie wirft dabei Andrea vor, die Dinge falsch dargestellt zu haben (Z. 06). Andrea verzichtet aber auf eine weitere Verlängerung der Streitsequenz und schließt diese stattdessen mit einem prosodisch unmarkierten, intonatorisch fallenden gut. ab. Dieses gut drückt keine Zustimmung oder gar positive Bewertung aus; es signalisiert vielmehr, dass man die Dinge ,so stehen lassen“ möge, wie sie sich bisher entwickelt haben.

Bisher wurde gezeigt, dass gut in responsiver, alleinstehender Verwendung seine bewertende Semantik verliert und stattdessen höchstens noch Zustimmung, oft aber lediglich Sequenzabschluss und (wie in Ausschnitt 23) Übergang in die nächste Aktivität signalisiert. Die Parallele zu genau ist offensichtlich. Auch dort wurde ja argumentiert, dass die Funktion, eine spezifische Form der Zustimmung auszudrücken (nämlich eine, die nicht in der Interaktion selbst hergestellt wurde, sondern schon vorgängig existiert hat), von einer zweiten überlagert und immer mehr abgelöst wird, die lediglich den Übergang zwischen zwei Aktivitäten markiert. Die Position des Markers (seine Diskurssyntax) verän- 
dert sich dabei. Er wird von einem responsiven Ausdruck zu einem übergangsmarkierenden. ${ }^{11}$

Gut hat allerdings noch eine andere, sehr häufig belegte Verwendungsweise: es führt nach partieller Zustimmung ein Gegenargument ein (vgl. Willkop 1988: 149-155). Hier ist erneut die Position des Markers betroffen, aber in einer anderen, sehr viel spezifischeren Weise: der responsive Diskursmarker wird zu einem (zugleich) TCU-initialen. Er markiert also nicht den Übergang zwischen verschiedenen Sequenzen, Aktivitäten oder Themen, sondern präziser den Übergang vom Argument des anderen Teilnehmers zum Gegenargument des neuen Sprechers.

\section{Beispiel (26) 'Fluchthilfe' (DOMIAN 15-11-19)}

((Ch. erzählt von ihrer Flucht aus der DDR, die dadurch notwendig wurde, dass sie sich in einen Westdeutschen verliebt hatte, den sie in der DDR beim Tanzen kennengelernt hatte.))

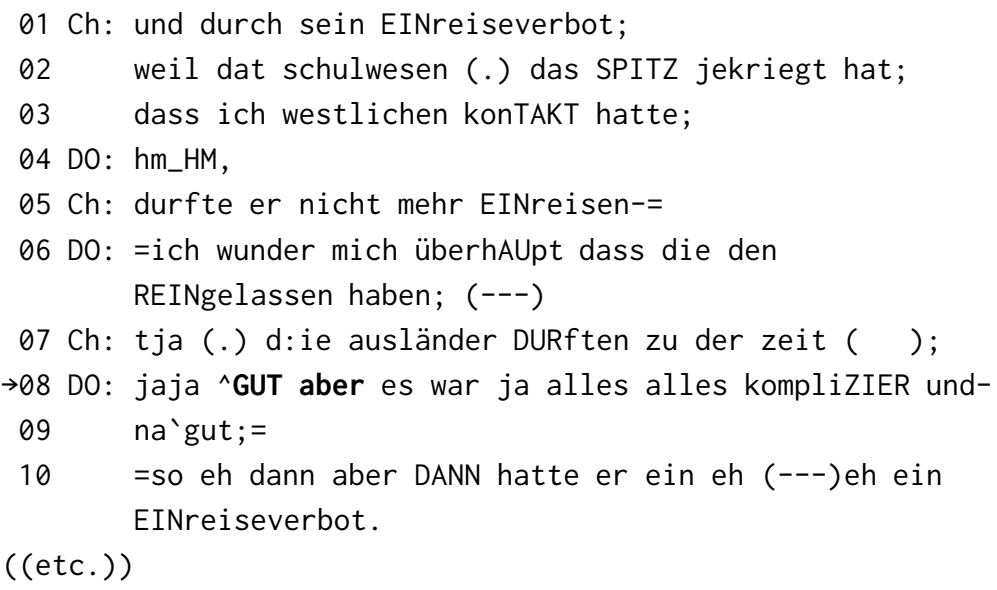

Formal unterscheidet sich dieses gut von den bisher diskutierten Verwendungen des Diskursmarkers durch seine relative prosodische Prominenz, die es im Kontext nach der oft vorlaufenden, unbetonten Partikel (na)ja erhält. Der Akzent auf GUT wird steigend-fallend realisiert. Funktional ist diese Verwendung dadurch gekennzeichnet, dass der Sprecher ein Argument der anderen Teilnehmerin (vorläufig) akzeptiert, es aber nicht für relevant oder ausschlaggebend hält. In Ausschnitt (26) hat zum Beispiel Domian in Z. 06 seiner Verwunderung darüber Ausdruck gegeben, dass Ch.s westdeutscher Freund überhaupt in die DDR einreisen durfte. Ch. erwidert darauf, dass Westdeutsche grundsätzlich in die DDR einrei-

11 Vgl. dazu auch die ähnliche Entwicklung von span. bueno, die Raymond (2018) beschreibt. 
sen durften (im Gegensatz zu DDR-Bürgern, denen die Ausreise in die BRD in der Regel nicht gestattet war). Domian akzeptiert in Z. 08 diese Berichtigung zwar (mit jaja ^GUT), führt aber zur Verteidigung seiner Meinung (und Begründung seiner Verwunderung) an, dass es doch ,kompliziert‘ gewesen sei. ${ }^{12}$

Die für Gegenargumente typische Verwendung des Diskursmarkers gut hat sich ihrerseits zu einem Mittel zur monologischen Strukturierung komplexer Redebeiträge weiterentwickelt. Dabei steht (ja/na) gut meistens vor der Proposition, die konzediert wird, d. h. es hat sich eine weitere positionelle Veränderung ergeben:

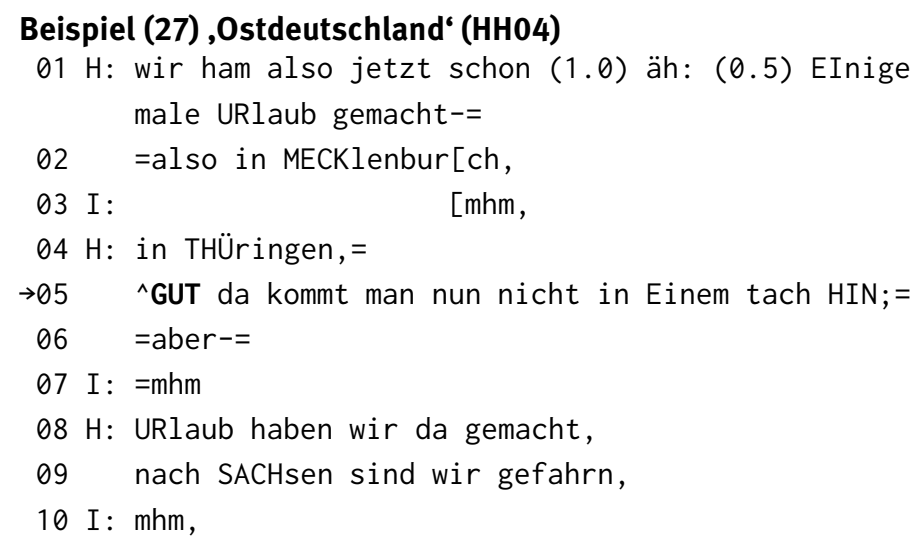

Der Sprecher, der von seinen Besuchen in den neuen Bundesländern berichtet, die er von Hamburg aus unternommen hat, baut eine konzessive Struktur auf: obwohl man (von Hamburg aus) nach Thüringen keine Tagesausflüge machen kann (wie in das vorher genannte Mecklenburg), hat er mit seiner Frau dort und in Sachsen bereits Urlaub gemacht. Der Redebeitrag ist nicht als Argument gegen den anderen Teilnehmer (I) strukturiert. Vielmehr enthält er selbst sowohl ein potenzielles Gegenargument gegen Reisen in die neuen Bundesländer (,nach Thüringen kommt man nicht in einem Tag') als auch die trotz dieser Tatsache geltende Aussage, wir haben in Thüringen Urlaub gemacht‘. Der Sprecher führt also eine konzessive Argumentation mit sich selbst. Wir haben es also erneut mit einem auto-reflexiven (autodialogischen) Gebrauch zu tun.

Noch klarer wird dies am folgenden Beispiel, in dem die interne Reflexion durch das Verb denken gekennzeichnet ist:

12 Im Ausschnitt kommt in Z. 09 ein weiteres, sequenzschließendes (na) gut vor. Man beachte die unterschiedliche Prosodie. 


\section{Beispiel (28) ,Jetlag' (FR7)}

((Sprecherin F. nimmt Melatonin gegen Jetlag.))

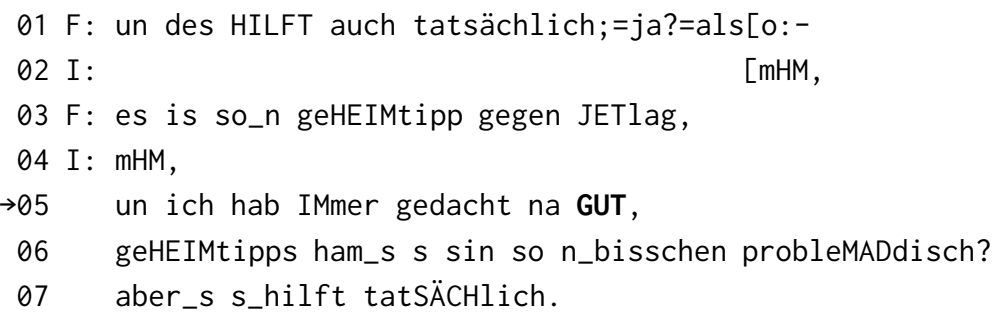

Auch hier ist die Gesamtstruktur konzessiv: ,obwohl Geheimtipps an sich problematisch sind', hilft Melatonin ,tatsächlich gegen Jetlag'. Wieder ist gut nicht hetero-dialogisch, sondern räumt die Gültigkeit eines Gegenarguments ein, das die Sprecherin selbst vorträgt, um dann auf ihrer Einschätzung des ,Geheimtipps‘ gegen Jetlags zu insistieren.

Interessanterweise spielt also auch bei der Entwicklung von gut (wie bei der von genau) der auto-reflexive Dialog eine Rolle. In Fall von gut lassen sich die folgenden Phasen der Entwicklung zum Diskursmarker rekonstruieren:

Ausgangspunkt: responsive Verwendung, alleinstehend

Sprecherin A: behauptet „p“

Sprecherin B: stimmt zu (gut) (,„“ ist im Skopus von gut)

Schritt 1: responsive TCU-initiale Verwendung

Sprecherin A: behauptet „p“

Sprecherin B: stimmt zu (gut) (,„”“ ist im Skopus von gut)

Sprecherin B: behauptet „aber q“ (wobei q ein Argument gegen p ist oder seine Gültigkeit zumindest einschränkt)

Schritt 2: turninterne Verwendung - anaphorisch (hier nicht besprochen)

Sprecherin B: behauptet „p“

Sprecherin B: stimmt sich selbst zu (gut) (,p“ ist im Skopus von gut)

Sprecherin B: behauptet „aber q“ (wobei q ein Argument gegen p ist oder seine Gültigkeit zumindest einschränkt)

Schritt 3: turninterne Verwendung - kataphorisch

Sprecherin B: stimmt sich selbst zu (gut) (,p“ ist im Skopus von gut)

Sprecherin B: behauptet „p“

Sprecherin B: behauptet ,aber q“ (wobei q ein Argument gegen p ist oder seine Gültigkeit zumindest einschränkt)

Allerdings ist die Rolle der auto-reflexiven Dialogizität eine andere als im Fall von genau. Dieses indiziert als Teil eines Selbstgesprächs die Bewältigung einer kognitiven Aufgabe, während jenes die Gültigkeit eines (möglichen) Gegenarguments 
konzediert. Und während bei genau die auto-reflexive Komponente essentiell für die Entwicklung des Markers zu einem übergangsmarkierenden Gliederungssignal ist, spielt sie bei gut erst eine Rolle, wenn der Marker sich vom alleinstehenden oder turn-initialen Abschluss- bzw. Übergangsmarker zum einleitenden Element einer konzessiven Formulierungspraktik entwickelt, die schon wieder den Weg zurück von der Diskurs- zur Satzsyntax einleitet.

\section{Abschließende Bemerkungen}

Genau! In einer Powerpoint-Präsentation könnte der Übergang zur Folie mit den abschließenden Bemerkungen so eingeleitet werden. Für diejenigen von uns, die dieses genau nicht mehr auffällig finden, hat sich der Diskursmarker von seiner ursprünglichen Funktion gelöst und ist zu einem rein formalen, übergangmarkierenden Marker geworden, vergleichbar mit gut oder ok in derselben Position.

Ich habe in diesem Beitrag versucht, diese Entwicklung zu rekonstruieren. Die ursprüngliche Funktion des sequenziell responsiven genau wurde dabei (in Übereinstimmung mit Willkop 1988 und Oloff 2017) nicht in der einfachen $\mathrm{Zu}$ stimmung gesehen, sondern im Ausdruck der Übereinstimmung von Meinungen, Absichten oder Wissensbeständen, die als ,immer schon` vorhanden dargestellt wird.

Die Analyse ergab, dass, von dieser Funktion ausgehend, der Übergang zum Gliederungssignal auf zwei Mechanismen beruht, die für die Entwicklung von Diskursmarkern von allgemeinerer Bedeutung sind. Den einen könnte man, wenn man Paradoxien liebt, als Dialogisierung des Monologs (oder Monologisierung des Dialogs) beschreiben. Statt als Teil einer interaktiven Sequenz, in der genau der Meinung, der Absicht oder dem Wissen des Anderen zustimmt, bestätigt der Sprecher sich selbst und führt dem Rezipienten die Bewältigung eines kognitiven Problems vor. Solche auto-reflexiven Dialoge spielen (in anderer Funktion) auch bei konzessiven monologischen Turnkonstruktionen mit den Zustimmungspartikeln gut (und auch schön, klar, ok) eine Rolle. Dort haben sie allerdings nichts mit der Bewältigung kognitiver Aufgaben zu tun, sondern dienen der Externalisierung eines internen, argumentativen Dialogs des Sprechers mit sich selbst. Der zweite Mechanismus ist ein Wandel der ,Makrosyntax، (Gülich 1970). Ein Diskursmarker, der ursprünglich sequenziell responsiv (und von daher sequenzterminierend) verwendet wird, nimmt (zusätzlich) eine einleitende Funktion an und wird so zum Gliederungsmarker. Auch dieser Prozess ist nicht nur bei genau, sondern auch bei anderen Zustimmungsmarkern zu beobachten. Er wird dort aber in anderen Phasen und mit anderen Ergebnissen relevant. 
Ich habe die Diskussion des neuen Diskursmarkers genau in der Funktion eines Übergangsmarkers mit einem Beispiel aus einer Powerpoint-Präsentation begonnen und in diesem Zusammenhang von einem Power-Point-Genau gesprochen. Die Häufigkeit, mit der der Diskursmarker in dieser mündlichen Gattung vorkommt, scheint eine solche Bezeichnung zu rechtfertigen. Nun baut die PPP natürlich auf älteren Formen des ,Vortrags` auf, etwa der durch projizierte Dias oder (später) der durch Folien auf Tageslichtprojektoren unterstützten Präsentation. Dass allerdings auch schon innerhalb solcher Präsentationen Übergänge vom Sprecher mit genau markiert worden wäre, habe ich nicht in Erinnerung. Von Dias unterscheidet sich die PPP dadurch, dass jene nicht mehr als illustrierende Funktion hatten, nie aber die Struktur des Vortrags visualisierten. Von der OverheadFolie unterscheidet sich die PPP vermutlich vor allem dadurch, dass die Interaktion mit dem Computer dem Präsentator wesentlich weniger Freiheit einräumt. Die PPP auf dem Rechner ist ja im Augenblick der Präsentation selbst nicht mehr zu verändern, selbst Änderungen der Reihenfolge der Folien bleiben dem Publikum nicht verborgen. Wer eine PP präsentiert, muss mit dem umgehen, was er oder sie vorher festgelegt hat (und sich nicht zuletzt daran erinnern). Der Tageslichtprojektor überließ es den Vortragenden, ob und in welcher Reihenfolge sie die Folien auflegen wollten (und oft wurden die Folien handschriftlich während der Präsentation beschrieben oder verändert). Die kognitiven Herausforderungen, die PP dem Präsentator abverlangt, sind also ungleich höher und die Notwendigkeit, sich selbst die erfolgreiche Bewältigung dieser Herausforderungen zu bestätigen, ist entsprechend gestiegen. ${ }^{13}$

Aber übergangsmarkierendes genau kommt heute natürlich nicht (mehr?) nur in dieser Gattung vor. Neue Medien, neue Software und die in ihrer Folge entstandene mündliche Gattung der PP-Präsentation können die Sprache verändern; zu fragen ist aber, wie die Beziehung zwischen der neuen Gattung und dem Wandel des Diskursmarkers zu modellieren ist. Eine empirisch begründete Antwort lässt sich im vorliegenden Fall nicht geben, es gibt aber zumindest zwei sehr plausible Szenarios. Im einen treibt die massenhafte, für viele Menschen alltägliche Verwendung einer Gattung wie der PPP einen schon in Gang befindlichen Wandelprozess an, indem sie als Katalysator wirkt und die neue Entwicklung beschleunigt. Im anderen Szenario schafft die neue Gattung erst den Kontext, in dem der sprachliche Wandel motiviert wird. Beide Szenarios sind denkbar und belegen, dass das Konzept der mündlichen Gattung für die Erklärung des Sprachwandels eine wichtige Rolle spielt.

13 Die Software-Entwickler haben darauf reagiert, indem sie in neueren PP-Versionen erlauben, die nächste Folie bereits während der Präsentation der vorherigen zumindest zu sehen und sich so auf sie einstellen zu können. 


\section{Literatur}

Antaki, Charles, Hanneke Houtkoop-Steestra und Mark Rapley (2000): 'Brilliant. Next Question' High-Grade Assessment Sequences in the Completion of Interactional Units. In: Research on Language and Social Interaction, 33(3):235-262.

Auer, Peter und Susanne Günthner (2005): Die Entstehung von Diskursmarkern im Deutschen ein Fall von Grammatikalisierung? In: Leuschner, Torsten, Tanja Mortelmans und Sarah de Groodt (Hrsg.), Grammatikalisierung im Deutschen, S. 335-362. Berlin: de Gruyter.

Auer, Peter und Jan Lindström (2016): Left/right asymmetries and the grammar of pre- vs. postpositioning in German and Swedish talk-in-interaction. In: Language Sciences, 56:68-92. (doi:10.1016/j.langsci.2016.03.001).

Auer, Peter und Anja Stukenbrock (2018): When 'You' Means 'l': The German 2nd Ps. Sg. Pronoun du between Genericity and Subjectivity. In: Open Linguistics, 4(1):280-309. (https://doi.org/10.1515/opli-2018-001).

Barth-Weingarten, Dagmar (2014): Dialogism and the emergence of final particles. The case of and. In: Günthner, Susanne, Wolfgang Imo und Jörg Bücker (Hrsg.), Grammar and Dialogism, S. 335-366. Berlin: de Gruyter.

Brenning, Jana (2015): Syntaktische Ko-Konstruktionen im gesprochenen Deutsch. Heidelberg: Winter.

Deppermann, Arnulf (2007): Deontische Infinitivkonstruktionen: Syntax, Semantik, Pragmatik. Berlin: de Gruyter.

Deppermann, Arnulf (i. d. B.): Imperative im Deutschen: Konstruktionen, Praktiken oder social action formats? In: Weidner, Beate et al. (Hrsg.), Verfestigungen in der Interaktion. Konstruktionen, sequenzielle Muster, kommunikative Gattungen. Berlin: de Gruyter.

Deppermann, Arnulf und Axel Schmidt (2016): Partnerorientierung zwischen Realität und Imagination: Anmerkungen zu einem zentralen Konzept der Dialogtheorie. In: Zeitschrift für Germanistische Linguistik, 44(3):369-405.

Drake, Veronika (2016): German questions and turn-final oder. In: Gesprächsforschung, 17:168-195.

Golato, Andrea und Emma Betz (2008): German ach and achso in repair uptake: A resource to sustain or remove epistemic asymmetry. In: Zeitschrift für Sprachwissenschaft, 27:7-37.

Gülich, Elisabeth (1970): Makrosyntax der Gliederungssignale im gesprochenen Französisch. München: Fink.

Günthner, Susanne (2012): Eine interaktionale Perspektive auf Wortarten: das Beispiel und zwar. In: Rothstein, Björn (Hrsg.), Nicht-flektierende Wortarten, S. 14-47. Berlin: de Gruyter.

Günthner, Susanne (2015): Ko-Konstruktionen im Gespräch: Zwischen Kollaboration und Konfrontation. In: Dausendschön-Gay, Ulrich, Elisabeth Gülich und Ulrich Krafft (Hrsg.), KoKonstruktionen in der Interaktion. Die gemeinsame Arbeit an Äußerungen und anderen sozialen Ereignissen, S. 55-74. Bielefeld: transcript Verlag.

Günthner, Susanne und Hubert Knoblauch (2007): Wissenschaftliche Diskursgattungen PowerPoint et al. In: Auer, Peter und Harald Baßler (Hrsg.), Reden und Schreiben in der Wissenschaft, S. 53-65. Frankfurt a. M.: Campus.

Heritage, John und Marja-Leena Sorjonen (2018): Introduction: Analyzing turn-initial particles. In: Heritage, John und Marja-Leena Sorjonen (Hrsg.), Between Turn and Sequence. TurnInitial Particles Across Languages, S. 1-22. Amsterdam: Benjamins. 
Knoblauch, Hubert (i. d. B.): Von kommunikativen Gattungen zu kommunikativen Formen: Konsequenzen des kommunikativen Konstruktivismus. In: Weidner, Beate et al. (Hrsg.), Verfestigungen in der Interaktion. Konstruktionen, sequenzielle Muster, kommunikative Gattungen. Berlin: de Gruyter.

König, Ekkehard (1991): Identical values in conflicting roles: the use of German ausgerechnet, eben, genau and gerade as focus particles. In: Abraham, Werner (Hrsg.), Discourse Particles, S. 11-36. Amsterdam: Benjamins.

Lehmann, Christian (1985/2002): Thoughts on Grammaticalization. Zweite, revidierte Fassung. Arbeitspapier 9, Seminar für Sprachwissenschaft der Universität Erfurt. online (pdf).

Linke, Angelika (i. d. B.): Musterwandel als Indikator für soziokulturellen Wandel. Ein Abriss zur Veränderung von Grussformeln vom 17. zum 21. Jahrhundert. In: Weidner, Beate et al. (Hrsg.), Verfestigungen in der Interaktion. Konstruktionen, sequenzielle Muster, kommunikative Gattungen. Berlin: de Gruyter.

Oloff, Florence (2016): Comparaison de deux marqueurs d'affirmation dans des séquences de co-construction: voilà et genau. In: Testi e Linguaggi, 10:243-267.

Oloff, Florence (2017): Genau als redebeitragsinterne, responsive, sequenzschließende und sequenzstrukturierende Bestätigungspartikel im Gespräch. In: Blühdorn, Hardarik et al. (Hrsg.), Diskursmarker im Deutschen, S. 207-232. Göttingen: Verlag für Gesprächsforschung.

Raymond, Chase Wesley (2018): Bueno-, pues-, and bueno-pues-prefacing in Spanish conversation. In: Heritage, John und Marja-Leena Sorjonen (Hrsg.), Between Turn and Sequence. Turn-Initial Particles Across Languages, S. 59-96. Amsterdam: Benjamins.

Schegloff, Emanuel (1996): Turn organization: One intersection of grammar and interaction. In: Ochs, Elinor, Emanuel Schegloff und Sandra A. Thompson (Hrsg.), Interaction and Grammar, S. 52-133. Cambridge: CUP.

Stukenbrock, Anja (2010): Überlegungen zu einem multimodalen Verständnis der gesprochenen Sprache am Beispiel deiktischer Verwendungsweisen des Ausdrucks so. In: InLiSt Interaction and Linguistic Structures, 47. http://www.inlist.uni-bayreuth.de/issues/47/ InLiSt47.pdf (18.08.2020).

Werlen, Iwar (2010): Genau als Hörersignal im (Schweizer-)Deutschen. In: Harden, Theo und Elke Hentschel (Hrsg.), 40 Jahre Partikelforschung, S. 345-359. Tübingen: Stauffenburg. Willkop, Eva-Maria (1988): Gliederungspartikeln im Dialog. München: iudicium. 